\title{
RESEARCH PAPER RP1647
}

\author{
Part of Journal of Research of the National Bureau of Standards, Volume 34, \\ April 1945
}

\section{FREEZING TEMPERATURE OF BENZOIC ACID AS A FIXED POINT IN THERMOMETRY}

\author{
By Frank W. Schwab and Edward Wichers
}

\section{ABSTRACT}

The freezing temperature of benzoic acid is found to be reproducible with a precision comparable to that of the ice point and somewhat superior to that of the steam point as usually observed in standardizing laboratories. For use in the calibration of thermometric instruments the acid is contained in a partially evacuated glass cell provided with a thermometer well. When thus confinea, and if not heated excessively, the substance maintains a constant composition for long periods of time. When properly manipulated, the acid in a given cell exhibits a freezing temperature reproducible from day to day within 1 millidegree.

The triple point of pure benzoic acid is $122.362^{\circ} \pm 0.002^{\circ} \mathrm{C}$. Under a pressure of 1 atmosphere of dry air the freezing temperature is 0.013 degree higher. Corresponding elevations for oxygen and nitrogen are 0.001 and 0.015 degree, respectively. The pressure coefficient of the freezing temperature is 0.039 degree/atm. The change in volume of the acid on freezing is $-0.138 \mathrm{~cm}^{3} / \mathrm{g}$. The corresponding calculated value for the density of the solid at the freezing temperature is 1.27 $\mathrm{g} / \mathrm{cm}^{3}$. The solubility of oxygen in the liquid at its freezing temperature, under a pressure of $1 \mathrm{~atm}$., is $0.132 \mathrm{~g} / 1,000 \mathrm{~g}$ of acid; that of nitrogen is $0.073 \mathrm{~g}$.

An appendix deals with a method of calculating temperatures, in the ranges $-190^{\circ}$ to $0^{\circ} \mathrm{C}$ and $0^{\circ}$ to $660^{\circ} \mathrm{C}$, from observations with a platinum thermometer. For this purpose power-series equations are used instead of the Callendar and Callendar-Van Dusen equations. Tables are included to facilitate the computation of temperature.

\section{CONTENTS}

I. Introduction

II. Materials, apparatus, and procedures_. 335

1. Material and procedure for filling the cells

2. Apparatus for measuring temperatures_._. _ _ _ _ _ _ _ 339

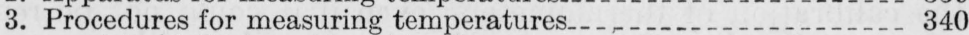

(a) At the benzoic acid point

(b) At the ice point

III. Stability of benzoic acid p....... 342

IV. Reproducibility of the freezing temperature

1. Relative reproducibility of the ice point and the benzoic acid point__.

2. Comparison of thermometers

3. Comparison of cells

V. Freezing temperatures of the pure acid under defined conditions

1. Determination of the triple point

2. Freezing temperatures under different pressures of oxygen and of nitrogen

3. Variation in the freezing temperature as a function of pressure..- 354

4. Solubilities of oxygen and of nitrogen in benzoic acid_...... 355

5. Freezing temperature under one atmosphere of dry air...... 356

VI. Change in the specific volume of benzoic acid that accompanies freezing -359 
VII. Appendix. Calculation of temperatures in platinum resistance thermometry (by Frank W. Schwab and E. R. Smith)
1. Relation between the power series, Callendar and Callendar-

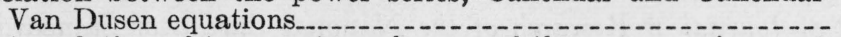

2. Interpolation of temperatures by use of the power series equations............... 361

(a) In the range from 00 to $660^{\circ} \mathrm{C}^{\circ}$

(b) In the range from $-190^{\circ}$ to $0^{\circ} \mathrm{C}$

3. Procedure for the calculation of temperatures by use of the

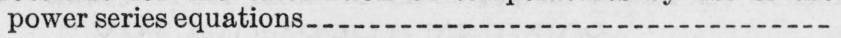

\section{INTRODUCTION}

Temperatures in the range $0^{\circ}$ to $660^{\circ} \mathrm{C}$, on the International Temperature Scale, are defined in terms of platinum resistance thermometers, of specified characteristics, by means of the equation

$$
R_{t}=R_{0}\left(1+A t+B t^{2}\right) .
$$

$R_{0}$ is the resistance at the temperature of equilibrium between ice and air-saturated water at normal atmospheric pressure, and $A$ and $B$ are constants determined by calibration at the temperatures of condensing water vapor and sulfur vapor, respectively, each under a pressure of 1 atm. The temperature of melting ice is relatively insensitive to variations of atmospheric pressure $(0.0076$ degree C/atm.), but the accuracy of calibration at the steam and sulfur points is limited by the accuracy which can be attained in making barometric measurements. The boiling temperature of water changes by 0.001 degree $C$ with a change in pressure of $0.027 \mathrm{~mm}$ of mercury. Since barometric pressure measurements commonly are not made to an accuracy greater than about $0.1 \mathrm{~mm}$ of mercury in standardizing laboratories, the accuracy of calibration of platinum thermometers is limited to 0.003 to 0.004 degree C, except in a very few laboratories that are equipped for fundamental work in thermometry. The sulfur point, used with the steam and ice points to determine the constant in the second-power term in the equation, need not be fixed as accurately as the steam point unless temperatures are measured near the sulfur point or in the range beyond it.

A readily reproducible fixed temperature in the vicinity of $100^{\circ} \mathrm{C}$, insensitive to variations in barometric pressure, would be useful for the calibration of thermometers, even if it could not be reproduced more exactly than the steam point, because the calibration procedure would be greatly simplified if barometric measurements did not need to be made. The present paper deals with the use for this purpose of the freezing temperature of benzoic acid. The term "freezing temperature" is here used in preference to "freezing point" because, as will be explained later, it is proposed to establish arbitrary conditions of observation, which may cause the freezing temperature to be fixed anywhere between the triple point $\left(122.362^{\circ} \mathrm{C}\right)$ and the freezing point (freezing temperature under $1 \mathrm{~atm}$. of dry air, $122.375^{\circ} \mathrm{C}$ ).

Experience with benzoic acid in another project ${ }^{1}$ indicated that this substance possesses the properties required of a thermometric standard, of which the more important are as follows: (1) Availa-

1 Frank W. Schwab and Edward Wichers, Preparation of benzoic acid of high purity, J. Research NBS $\mathbf{2 5}$, 747 (1940) RP1351. 
bility in an adequate degree of purity; (2) sufficiently large latent heat at the change of phase; (3) adequate velocity of crystallization; (4) existence in only one crystalline form at temperatures near the freezing point; and (5) stability under the conditions of use. Benzoic acid can be obtained commercially in a degree of purity better than 99.95 mole percent, and the purity can be increased to 99.998 mole percent or better by relatively simple laboratory procedures, most readily by a method of fractional freezing described in a previous paper. ${ }^{2}$ The cryoscopic behavior of the substance is such that 0.001 mole percent of impurity dissolved in the liquid acid (but insoluble in the solid) depresses the freezing temperature 0.00075 degree C. Hence the attainable purity will permit reproducing the freezing temperature to within 0.001 to 0.002 degree C. The same amount of impurity causes the freezing temperature of the acid to change 0.00075 degree $\mathrm{C}$ between 0 - and 50-percent frozen. As will be shown later, variations in the proportion frozen can be held within a small part of this range.

The heat of fusion of benzoic acid is reported to be about $140 \mathrm{j} / \mathrm{g}$, or $150 \mathrm{j} / \mathrm{ml}$, which is a little less than half that of water. Many observations made during earlier work show that the heat of fusion, combined with a high velocity of crystallization, is sufficient to maintain satisfactory thermal equilibrium between the solid and liquid when the system loses heat at any reasonably slow rate. During these observations no evidence was ever obtained of the transformation of the liquid to more than one crystalline form.

\section{MATERIALS, APPARATUS, AND PROCEDURES}

\section{MATERIAL AND PROCEDURE FOR FILLING THE CELLS}

The benzoic acid used for the experimental work reported in this paper was prepared by fractionally freezing a quantity of commercial benzoic acid as described by the authors in an earlier publication. ${ }^{3}$ Measurements of the freezing range, ${ }^{4}$ when the acid was freed of water in a manner which did not cause the formation of a significant amount of benzoic anhydride, indicated that the acid used to determine the reproducibility of the freezing temperature was 99.998 mole percent pure. That used to study some of the properties of the acid was of somewhat lower purity.

To avoid variations in freezing temperature which would have resulted from changes in the moisture content of air had the acid been frozen in open vessels, the acid was kept in sealed cells for all observations of the constancy and reproducibility of the freezing temperature. These cells, shown in figure 1 , were cylindrical in shape, $5 \mathrm{~cm}$ in diameter, and about $25 \mathrm{~cm}$ long. A thermometer well $8 \mathrm{~mm}$ in diameter extended nearly the length of the cylinder, and an extension of the well about $12 \mathrm{~cm}$ long served as a handle. The capacity of each cell was about $425 \mathrm{ml}$.

2 Frank W. Schwab and Edward Wichers, Purification of substances by slow fractional freezing, J. Research NBS 32, 253 (1944) RP1588.

3 See footnote 2.

- Frank W. Schwab and Edward Wichers, Precise Measurement of the Freezing Range as a Means of Determining the Purity of a Substance, Temperature-Its Measurement and Control in Science and Industry, p. 256-264 (Reinhold Publishing Corporation, New York, N. Y., 1941). 
Before the cell was sealed to the apparatus shown in figure 2 , about $380 \mathrm{~g}$ of the acid $(365 \mathrm{ml})$, carefully fused in a beaker or other suitable vessel and containing a small amount of water, was transferred to the cell through a side tube. The volume of liquid acid was adjusted so as to leave a free space of 50 to $60 \mathrm{ml}$ in the cell. The acid was allowed to freeze completely and the cell was sealed in place as shown

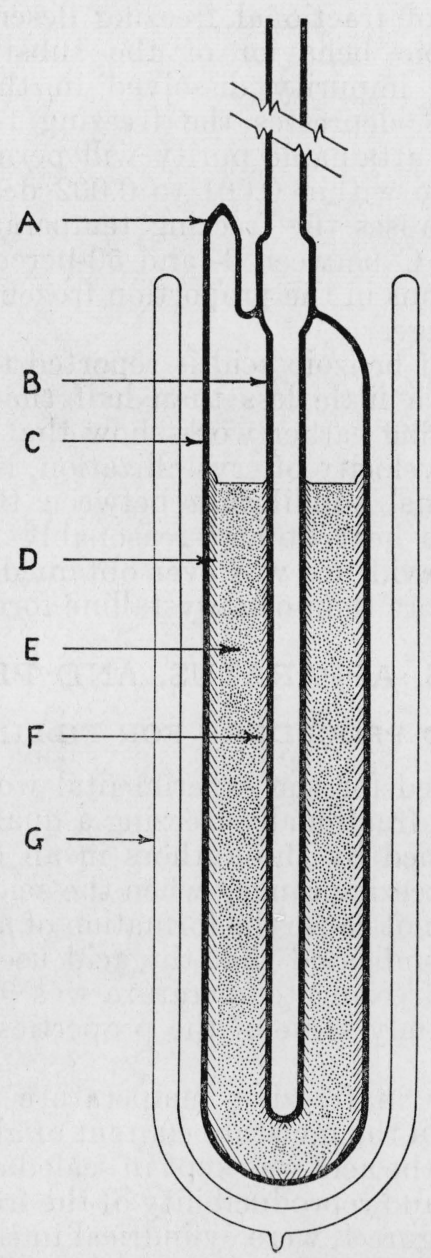

Figure 1.-Benzoic acid fixed-point cell.

$A$, Side-tube; $B$, thermometer well; $C$, cell proper; $D$, solid benzoic acid; $E$, mixture of crystals and liquid $F$, solid benzoic acid; $G$, Dewar flask.]

in figure 2 , with the aeration-tube, $B$, completely withdrawn. The acid was melted again and tube $B$ was inserted, through the sidearm extended, into the liquid and adjusted with its end near the bottom of the cell. The opening at $K$ between $B$ and the side arm extended was closed by forcing a short piece of rubber tubing over the outside tube and was finally sealed with pycene. With stopcock 9 open, 
and stopcock 2 open to outlet 1 , the liquid was saturated with air, which was dried over phosphorus pentoxide and free of carbon dioxide, forced under the necessary pressure into the system at $H$.

The next steps in the procedure were designed to remove the water which is always present when benzoic acid is fused in contact with ordinary air. Liquid benzoic acid saturated, at temperatures near its freezing point, with air having a partial pressure of $5 \mathrm{~mm}$ of water vapor, contains about 0.06 mole percent of dissolved water. This amount of water depresses the freezing temperature between 0.04 and 0.05 degree $\mathrm{C}$. In addition to moisture gained from the air there was

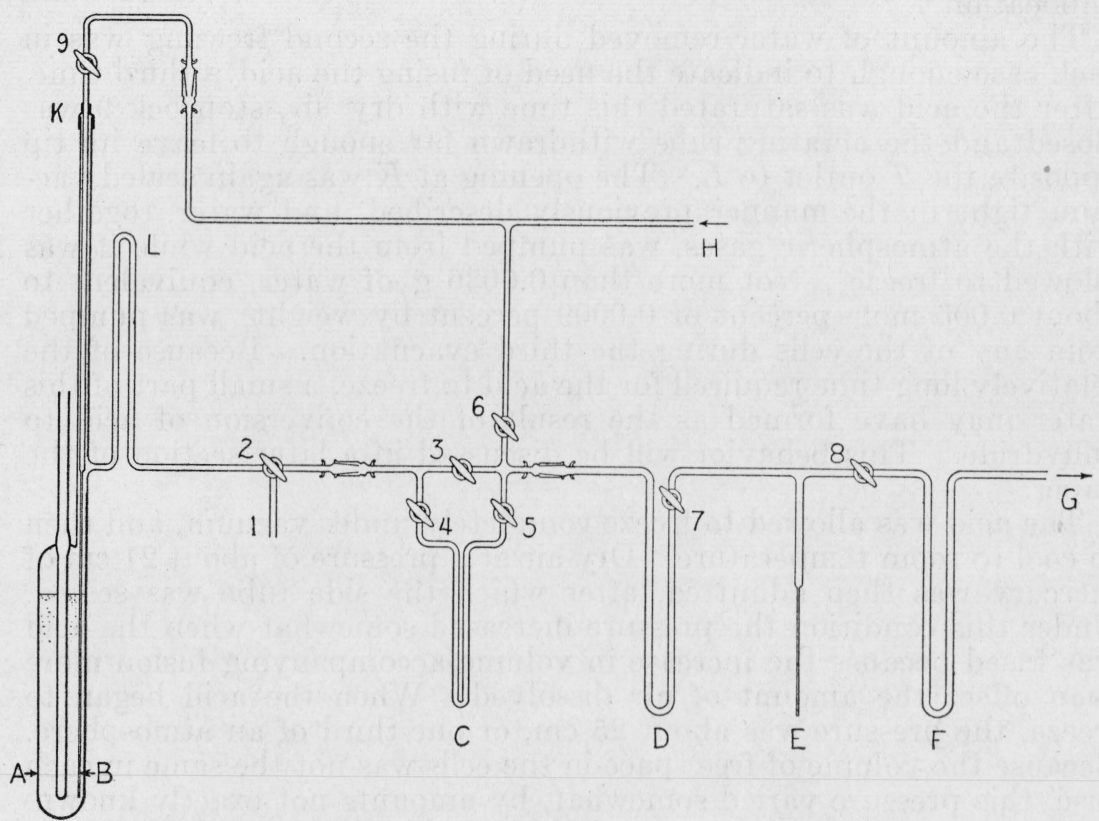

FiguRe 2.-Apparatus for filling the benzoic acid fixed-point cell and for determining water in benzoic acid.

$A$, Benzoic acid fixed-point cell; $B$, aeration-tube; $C$, manometer; $D$, trap of known volume; $E$, capillary trap; $F$, trap; $G$, vacuum connection; $H$, dry air source; $K$, rubber-tubing-connection sealed with pycene; $L$, air condenser for benzoic acid; 1 , outlet for dry air; 2,3 , 4, etc. 2-mm stopcocks.

usually some that had been added intentionally to convert to benzoic acid small amounts of benzoic anhydride which might have been formed during the operations of purification. It was therefore necessary to dry the acid in the cells quite thoroughly to avoid excessive differences among the freezing temperatures in the several cells. This was done by pumping off the gases evolved while the acid slowly froze in the cell. The evolution of dissolved oxygen and nitrogen helped materially to sweep out the water vapor. The acid was allowed to freeze completely. To keep it from freezing so rapidly as to entrap much of the evolved gases, it was warmed at intervals with the soft flame of a hand torch. The acid was again fused and saturated with dry air. During this operation much of the acid that had sublimed while it was previously freezing and had condensed in the cell side of the inverted $U$-tube, $L$, melted and flowed back into the cell. The 
acid was allowed to freeze a second time. This time the discharged gases were pumped off through stopcock 2 , which was open to the manometer, $C$, and trap $D$ with stopcock 6 closed. During the pumping the trap was cooled with liquid air. When the pumping was completed, the trap was isolated from the rest of the apparatus, by closing stopcocks 3 and 7, and allowed to warm to room temperature. The known volume of the trap and the pressure indicated by the manometer gave a measure of the amount of water removed from the acid. $E$ is a capillary receiver not used in this work and $F$ is a guard trap. This apparatus and its use are described in greater detail in another publication. $^{5}$

The amount of water removed during the second freezing was in each case enough to indicate the need of fusing the acid a third time. After the acid was saturated this time with dry air, stopcock 9 was closed and the aerating tube withdrawn far enough to leave its tip opposite the $T$ outlet to $L$. The opening at $K$ was again sealed vacuum tight in the manner previously described, and water, together with the atmospheric gases, was pumped from the acid while it was allowed to freeze. Not more than $0.0036 \mathrm{~g}$ of water, equivalent to about 0.006 mole percent or 0.0009 percent by weight, was pumped from any of the cells during the third evacuation. Because of the relatively long time required for the acid to freeze, a small part of this water may have formed as the result of the conversion of acid to anhydride. This behavior will be discussed in a later section of the paper.

The acid was allowed to freeze completely under vacuum, and then to cool to room temperature. Dry air at a pressure of about $21 \mathrm{~cm}$ of mercury was then admitted, after which the side tube was sealed. Under this condition the pressure increased somewhat when the acid was fused because the increase in volume accompanying fusion more than offset the amount of air dissolved. When the acid began to freeze, the pressure was about $25 \mathrm{~cm}$, or one-third of an atmosphere. Because the volume of free space in the cells was not the same in each case, this pressure varied somewhat, by amounts not exactly known, in the group of cells prepared for observations of the freezing temperature.

Several other cells, filled in the manner described, were used for observations on the stability of the acid. Still another cell (fig. 4), to be described later, was provided with a side arm assembly consisting of a stopcock and a ball-joint connection so that various gases could be admitted at known pressures for observations on the effect of pressure and of dissolved atmospheric gases. The triple point of the acid was also measured in this same apparatus.

5 Frank W. Schwab and Edward Wichers, A physical method for determining residual water and other volatile materials in pure substances, J. Research NBS 33, 121 (1944) RP1600. 


\section{APPARATUS ${ }_{\mathbb{N}}^{7} F O R$ MEASURING TEMPERATURES}

The temperature measurements were made with platinum resistance thermometers of the coiled-filament type described by Meyers, ${ }^{6}$ with a resistance at $0^{\circ} \mathrm{C}$ of about $25 \mathrm{ohms}$. The outside diameter of the thermometers was about $7 \mathrm{~mm}$ and the length of the coiled filament was 3 to $3.5 \mathrm{~cm}$. The resistances were measured with a precision Mueller ${ }^{7}$ bridge of the type G-2, No. 8069, made by the Leeds \& Northrup Co. The apparatus was equipped with a switch that reversed the galvanometer current and thereby doubled the scale deflection caused by a given unbalance in the bridge. For convenience the bridge was connected to a four-place selector switch. The galvanometer had a sensitivity of $10 \mathrm{~mm} /$ microvolt, a period of not over 6 seconds, a critical external damping resistance of not less than 50 ohms and a coil resistance of $23.5 \mathrm{ohms}$. The optical arm of the galvanometer was $5.5 \mathrm{~m}$ long. With a reversing switch on the bridge, it provided a scale deflection of $32 \mathrm{~mm}$ for an unbalance of the bridge of $0.0001 \mathrm{ohm}$. It was thus readily possible to estimate changes in resistance to a few millionths of an ohm. All corrections were made to a millionth of an ohm in order to obtain an adequate measure of the reproducibility.

Before the series of measurements reported in this paper were made, the resistance bridge was calibrated by the Resistance Measurements Section of this Bureau. Two of the thermometers, 535 and 618 , together with the bridge, were calibrated at the steam point to 0.0001 degree C by C. S. Cragoe and H. F. Stimson of the Heat Measurements Section, with a precision manometer by means of which the height of the mercury column was fixed with an accuracy within 1 micron. The sulfur point was determined in the regular way by the Thermometry Section, also of this Bureau. A much less accurate calibration was required at the sulfur point than at the steam point, since all of the temperatures measured were close to $122.4^{\circ} \mathrm{C}$. At this temperature, errors arising from an error of calibration at the sulfur point are only about one-fiftieth of the error at the sulfur point itself. The bridge and each of the thermometers, 535 and 618 , were calibrated in the same way after the measurements were completed, to disclose, if possible, any changes in the resistancemeasuring equipment. The other thermometers, 515 and 587, were calibrated in the regular way by the Thermometry Section, before the measurements were begun. At the completion of the observations, theremometer 587 was also calibrated with the same accuracy as 535 and 618 . The characteristics of the four thermometers, as determined by these calibrations, are given in table 1 .

6 C. H. Meyers, Coiled-filament resistance thermometers, BS J. Research 9, 807 (1932) RP508.

7 E. F. Mueller, Precision Resistance Thermometry, Temperature-Its Measurement and Control In Science and Industry, p. 162 to 179 (Reinhold Publishing Corporation, New York, N. Y., 1941). 
TABLE 1.-Characteristics of thermometers

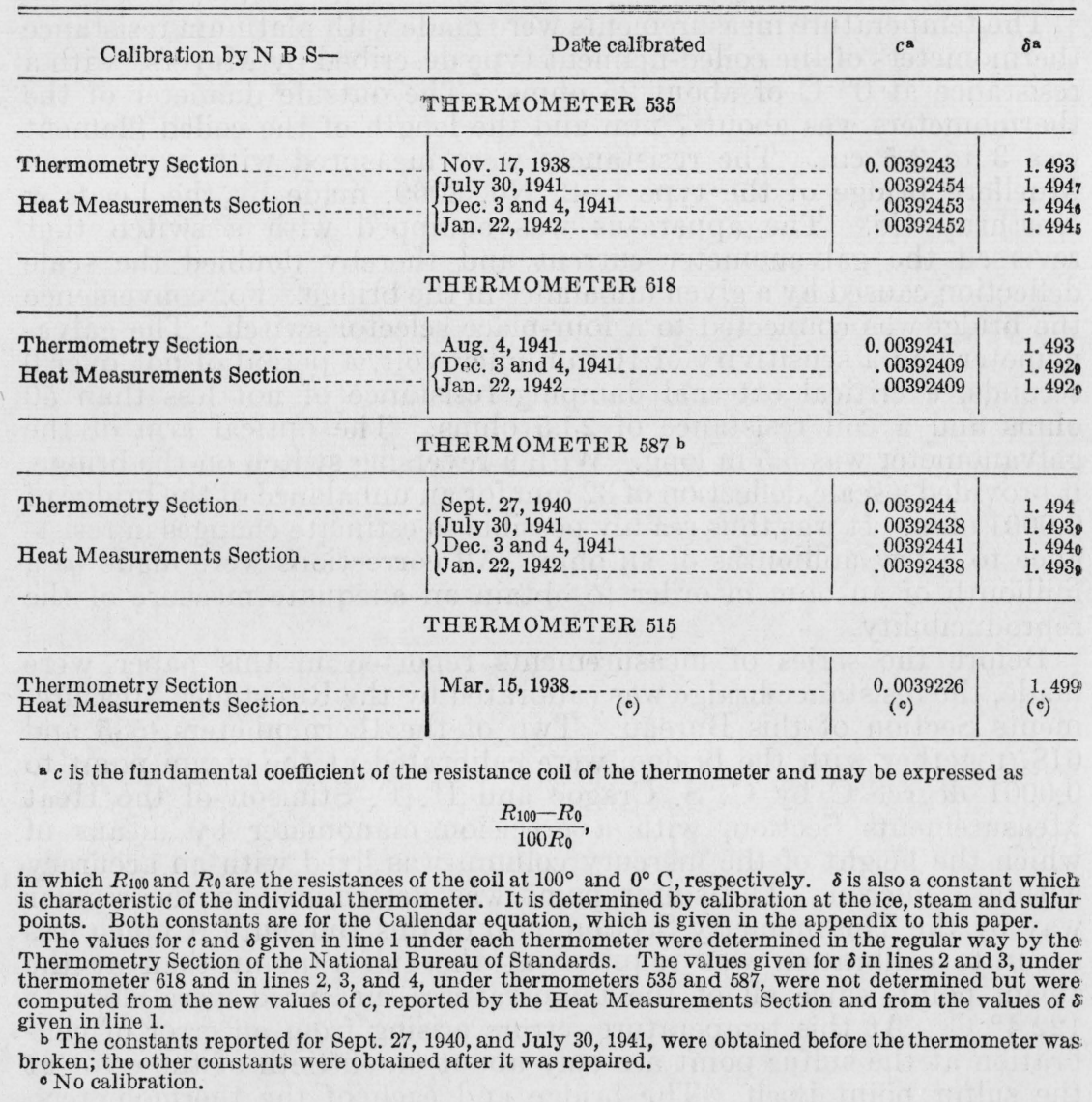

\section{PROCEDURES FOR MEASURING TEMPERATURES}

(a) AT THE BENZOIC ACID POINT

The procedure usually followed in observing the freezing temperatures in the cells was as follows: The cell was heated in an electric oven kept several degrees above the freezing point of the acid, usually at about $130^{\circ} \mathrm{C}$. When the acid was completely fused and had reached the temperature of the oven, the cell was removed and rather vigorously shaken so that the acid cooled uniformly. More rapid cooling at one point was caused by placing a cap of wet paper on the end of the side-tube ( $A$ in fig. 1 ). This caused a small amount of acid to crystallize and thus prevented excessive undercooling of the bulk of the liquid. If the crystals were not present, the acid sometimes undercooled as much as 15 degrees $\mathrm{C}$, and the amount of solid suddenly formed was excessive. Normally, as the cell cooled, a mush of fine needle-like crystals appeared quite suddenly throughout the liquid. Although only a small fraction of the acid was frozen, the crystals were so well interlaced that they did not tend to settle out on subsequent standing. The cell was then rolled up in a piece of soft cloth 
and placed in a close-fitting Dewar flask. The open end of the Dewar flask was packed with cotton to provide further insulation. Before the cell was put into it, the Dewar flask was warmed approximately to the freezing point of the acid by placing in it for 15 to 20 minutes a companion cell also containing freezing benzoic acid. This was done to prevent the rapid freezing of acid in the cell to be measured, which otherwise would have been caused by the loss of a considerable amount of heat to the cold flask. When the cell which was to be observed was in place in the Dewar flask, the resistance thermometer was inserted in the thermometer well. After about 10 minutes, measurements of resistance were begun. They were continued at least until the resistance reached its maximum (usually within 30 minutes) and nearly always for a considerably longer period. In a number of instances two sets of resistance measurements were made, one in which the thermometer current was $1.00 \mathrm{ma}$; the other, $1.41 \mathrm{ma}$. With the latter current the power input was twice that of the former. The difference in resistance between the two sets of measurements, when subtracted from the resistance observed with a current of $1 \mathrm{ma}$, provided the correction necessary to give resistances corresponding to zero thermometer current.

Usually, observations were made on several cells in close sequence by connecting the several thermometers to the bridge in rotation by means of the selector switch.

Temperatures corresponding to observed resistances were computed by the method described in the appendix to this paper. For observations of small differences in temperature, with a given thermometer, it was not always necessary to compute actual temperatures. For these relative measurements it was often sufficient to observe changes in the interval between the ice point and the benzoic. acid point, since the change of resistance as a function of temperature in the vicinity of the benzoic acid point was accurately known. The approximate coefficient was $0.0001 \mathrm{ohm} /$ millidegree.

\section{(b) AT THE ICE POINT}

The fundamental calibrations of the measuring instruments were made before and after the series of observations that gave the precision of the benzoic acid fixed-point cell. In addition, observations at the benzoic acid point were bracketed between observations at the ice point, made the same day, in order to disclose and, if possible, to correct for any variation in the resistance-measuring equipment. Two benzoic acid points and three ice points were usually measured with a thermometer during a day.

Measurements at the ice point were made as follows: ${ }^{8}$ Shaved ice obtained from clear cakes was washed with distilled water. The mush was thoroughly mixed in an open porcelain enamel pan to approach saturation with air, and then transferred to a precooled Dewar flask. The coil of the thermometer was immersed 20 to $25 \mathrm{~cm}$, the mush was pressed lightly around the thermometer and measurements

8 The "ice point," or temperature of equilibrium between ice and air-saturated water at normal atmospheric pressure, has been a basic fixed point in nearly all measurements of temperature. It was reported in the Technical News Bulletin of the National Bureau of Standards, No. 305, p. 71 (1942), that for work of the highest precision it is desirable to avoid the small variations in the ice point resulting from contact of the melting ice with the atmosphere. For this purpose the triple point of water has advantages. A direct comparison between the ice point and the triple point for the same sample of water shows a difference of 0.00997 degree $\mathbf{C}$. 
of the resistances were recorded when they became constant. As with the measurements at the benzoic acid point, observations were made with thermometer currents of 1.00 and $1.41 \mathrm{ma}$, to permit extrapolation to zero current. A correction for impurities in the ice was obtained from conductivity measurements of the ice water at the time of calibration and on other occasions when it was desirable to report temperatures to the highest accuracy. Clear ice was of high purity and the correction was usually not more than 0.00001 to $0.00002 \mathrm{ohm}$.

In table 5 are given the resistances of thermometers $587,535,618$, and 515 as measured at the ice points during the time most of the measurements reported in this paper were made. These measurements show the maximum variation observed during a period of about 1 month.

\section{STABILITY OF BENZOIC ACID}

In the course of this work, benzoic acid was not found to undergo any measurable irreversible decomposition when heated at temperatures below $150^{\circ} \mathrm{C}$. Evidence of this will be presented later. However, the acid does undergo a reversible decomposition to benzoic anhydride and water. Since this reaction must be controlled if the acid is not to suffer a variable or progressive change in purity, and hence in freezing temperature, extensive observations concerning it were made. Some of these observations have been reported in an earlier publication. ${ }^{9}$ When the acid is enclosed in a sealed container the water produced simultaneously with the anhydride cannot escape, and the reaction therefore can proceed only until an equilibrium is reached. At the outset it was thought that the acid would have to be brought to some state of equilibrium with the products of its own dissociation if reproducible freezing temperatures were to be obtained. Accordingly, four cells were kept above freezing temperature, except during the short periods when observations of the freezing temperature were being made, until they reached equilibrium, as evidenced by constancy of the freezing temperature. This was done by keeping the cells in an electrically heated oven, in which mechanical circulation of air kept the temperature constant within 1 degree. The temperature of thejoven was intended to be about 10 degrees $\mathrm{C}$ above the freezing temperature of the acid. An actual measurement made after the observations were begun showed the temperature of the oven to be $131.6^{\circ} \mathrm{C}$. The observed freezing temperatures of the cells are given in table 2. It will be noted that 10 days or longer were required for the acid to reach equilibrium with benzoic anhydride and water. The slight variability in the observed temperatures over longer periods, up to 8 weeks, is attributable to a lack of reproducibility caused by the presence of about 0.015 mole percent each of dissolved anhydride and water. The small differences in the total depressions observed in the several cells were probably caused by differences in the initial content of water or of anhydride resulting from uncontrolled variations in the drying of the acid when the cells were filled.

'Frank W. Schwab and Edward Wichers, Preparation of benzcic acid of high purity, J. Research NBS 25 , 747 (1940) RP 1351. 
TABLE:2.-Stability" of benzoic acid at $131.6^{\circ} \mathrm{C}$.

\begin{tabular}{|c|c|c|c|c|c|c|c|}
\hline \multicolumn{2}{|c|}{ Cell 11} & \multicolumn{2}{|c|}{ Cell 12} & \multicolumn{2}{|c|}{ Cell 13} & \multicolumn{2}{|c|}{ Cell 14} \\
\hline $\begin{array}{l}\text { Time at } \\
131.6^{\circ} \mathrm{C}\end{array}$ & $T_{p}$ & $\begin{array}{l}\text { Time at } \\
131.6^{\circ} \mathrm{C}\end{array}$ & $T_{\boldsymbol{P}}$ & $\begin{array}{l}\text { Time at } \\
131.6^{\circ} \mathrm{C}\end{array}$ & $T_{\boldsymbol{P}}$ & $\begin{array}{l}\text { Time at } \\
131.6^{\circ} \mathrm{C}\end{array}$ & $T_{F}$ \\
\hline$h r$ & ${ }^{\circ} C$ & & ${ }^{\circ} \mathrm{C}$ & $h r$ & & & \\
\hline & 122. 3577 & & 122. 3558 & 0 & 122. 3565 & & 122. 3598 \\
\hline & 122. 351 & 123 & 122. 344 & & - & & 122.356 \\
\hline & 122.347 & 161 & 122. 343 & 432 & 122.343 & $\begin{array}{l}58 \\
07 \\
07\end{array}$ & 122. 346 \\
\hline & 122. 341 & 265 & 122. 342 & $\begin{array}{l}560 \\
671\end{array}$ & $\begin{array}{l}12.342 \\
122.338\end{array}$ & 97 & 122.343 \\
\hline $\begin{array}{l}124 \\
143\end{array}$ & $\begin{array}{l}122.340 \\
122.341\end{array}$ & $\begin{array}{l}301 \\
383\end{array}$ & $\begin{array}{l}122.342 . \\
122.342\end{array}$ & 693 & 122.338 & 558 & $\begin{array}{l}122.339 \\
12.338\end{array}$ \\
\hline $\begin{array}{l}170 \\
161\end{array}$ & 122.340 & 419 & 122. 339 & 730 & 122.339 & 964 & 122.337 \\
\hline 180 & 122.341 & $\begin{array}{l}530 \\
530\end{array}$ & 122.341 & & & 1,075 & 122. 337 \\
\hline 247 & 122.338 & 548 & 122. 336 & - & 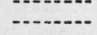 & & \\
\hline 265 & $\begin{array}{l}122.339 \\
\text { 12. }\end{array}$ & 566 & 122. 338 & - & - & - & - \\
\hline 284 & $\begin{array}{l}122.339 \\
1203\end{array}$ & $\begin{array}{l}602 \\
671\end{array}$ & $\begin{array}{l}122.340 \\
122.33\end{array}$ & - - - & -...-... & $\cdots$ & - \\
\hline $\begin{array}{l}302 \\
337\end{array}$ & $\begin{array}{l}122.339 \\
122.340\end{array}$ & $\begin{array}{l}671 \\
707\end{array}$ & $\begin{array}{l}122.338 \\
122.338\end{array}$ & - & - & - & - \\
\hline 405 & $\begin{array}{r}122.330 \\
\end{array}$ & 726 & $\begin{array}{l}122.338 \\
\end{array}$ & - & - & - & (n) \\
\hline 422 & $\begin{array}{l}122.339 \\
\end{array}$ & 1,073 & 122. 336 & - & - & - & - \\
\hline 525 & 122. 337 & 1,325 & 122. 337 & 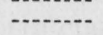 & - & - & - \\
\hline 579 & $\begin{array}{l}\text { 122. } 339 \\
123\end{array}$ & 1,391 & 122.337 & ........ & -......... & Nor & - \\
\hline 6882 & $\begin{array}{l}122.338 \\
122.373\end{array}$ & -....- & - n...- & -....... & - & -...- & 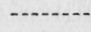 \\
\hline 995 & 122. 337 & - & - & - & -..... & - & 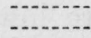 \\
\hline 1,061 & 122. 337 & - & - & - & 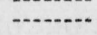 & - & - n...... \\
\hline $\begin{array}{l}1,281 \\
1,300\end{array}$ & $\begin{array}{l}122.336 \\
122.337\end{array}$ & ;....... & -...-. & a..... & - & :-o. & -.... \\
\hline $\begin{array}{l}1,318 \\
1,318\end{array}$ & $\begin{array}{l}122.338 \\
120337\end{array}$ & 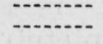 & ...... & ........ & - & $\cdots$ & - \\
\hline $\begin{array}{l}\text { After re- } \\
\text { version. }\end{array}$ & 122.3573 & $\begin{array}{l}\text { After re- } \\
\text { version. }\end{array}$ & 122.3549 & $\begin{array}{l}\text { After re- } \\
\text { version. }\end{array}$ & 122.3578 & $\begin{array}{l}\text { After re- } \\
\text { version. }\end{array}$ & 122.3600 \\
\hline
\end{tabular}

a In column 1 under each cell is given the total time in hours that the cell was kept at $131.6^{\circ} \mathrm{C}$. Proper allowance was made for each time the acid was frozen. The temperature of freezing $\left(T_{F}^{\circ} \mathrm{C}\right)$ represents the maximum temperature observed.

A few observations of the equilibrium established at $150^{\circ} \mathrm{C}$ showed that at this temperature the final concentrations of anhydride and water are approximately double those at $131.6^{\circ} \mathrm{C}$.

The last temperature recorded for each cell in table 2, designated "after reversion," indicates the freezing temperature after the completion of the other observations and after the original condition of the acid had been restored. It will be noted that in the long periods during which the cells were kept at $131.6^{\circ} \mathrm{C}$, no measurable irreversible decrease in the purity of the acid occurred. The average deviation of the freezing temperatures at the end of the several periods from those at the beginning was 0.7 millidegree.

Further evidence of the absence of thermal decomposition was given by the behavior of one cell that was kept at $131.6^{\circ} \mathrm{C}$ for nearly a year. The total depression of the freezing temperature observed during this interval was 5 millidegrees. Since the thermometer used to measure the freezing temperature at the beginning of the period was subsequently found to be variable by more than 5 millidegrees, the change that may have occurred is not precisely known, but it obviously was not large. The same cell was later kept at $150^{\circ} \mathrm{C}$ for nearly a month. After the first few days required to establish the anhydride equilibrium, there was no further depression of the freezing temperature, which would have occurred had there been a measurable amount of thermal decomposition or of a chemical reaction with the glass of the containing vessel. From these results it may be concluded that benzoic acid is adequately stable for use in thermometry, provided it is not heated for long periods at temperatures much above 
$150^{\circ} \mathrm{C}$, and provided account is taken of the reversible formation of benzoic anhydride and water.

During the observations made while the cells were kept at $131.6^{\circ} \mathrm{C}$, it was noted that the freezing temperature tended to rise if the acid was kept near freezing for longer than the usual short periods required for measurements. If all the acid in a cell was allowed to freeze slowly and then remelted for a short time, there was a marked rise in the freezing temperature. When this was repeated several times, the freezing temperature returned to that of acid containing no anhydride, as shown in table 2. Obviously the acid in the cells was reverting to its original condition. This came about for two reasons. From the fact that the equilibrium concentrations of anhydride and water at $150^{\circ} \mathrm{C}$ were markedly greater than those at $131.6^{\circ} \mathrm{C}$, it follows that the equilibrium concentrations near the freezing temperature $\left(122.4^{\circ} \mathrm{C}\right)$ must be lower than those at $131.6^{\circ} \mathrm{C}$. This alone is probably not enough to account for complete reversion to benzoic acid, although observations made at the freezing temperature indicate that the equilibrium concentrations of water and anhydride are lower than 0.003 mole percent each at this temperature. The probable explanation of complete reversion of the anhydride and water during slow freezing is as follows: As the acid freezes the anhydride and water remain almost completely in solution in the liquid phase. As freezing progresses, their concentrations in the liquid phase progressively increase. If the eutectic temperature of the system is as much as 10 degrees $\mathrm{C}$ below the freezing point of the acid, the initially minute concentration of the two "impurities" may be multiplied some thousands of times before the liquid phase completely disappears. This huge change in concentration causes the rate of recombination to increase enormously. It is doubtless true that the anhydride and water do not remain ideally in the liquid phase as freezing progresses, but this condition must be rather closely approached at the slow rate of freezing which occurs if the cell is kept in the Dewar flask.

From the data in table 2 it can be estimated that the initial rate of formation of anhydride and water at $131.6^{\circ} \mathrm{C}$ is not great enough to cause in 1 hour a lowering of the freezing temperature by as much as 1 millidegree. When a cell is prepared for an observation it takes only a short time, at most half an hour, to heat the acid, once it is completely fused, to a temperature a few degrees above its freezing point. As the rate of formation of the anhydride during the melting period, which is considerably longer, must be much lower than at $131.6^{\circ} \mathrm{C}$, it may be concluded that the total amount of anhydride and water formed while the cell is being prepared for an observation is not enough to depress the freezing temperature by as much as 1 millidegree. Since even the minute amounts that are formed tend to revert to acid during the subsequent freezing, it is apparent that cells kept at room temperature during the periods between observations would not be unreliable because of the dissociation reaction. Because of the absence of appreciable amounts of anhydride and water as "impurities" in such cells, the observed freezing temperatures would also be more sharply reproducible than in cells maintained above the freezing temperature during the intervals between observations. Accordingly, all subsequent observations of the reproducibility of the freezing tempera- 
ture were made on cells kept at room temperature when not in use. In occasional exceptions, for example, when the formation of crystals was faulty, the period required to reheat the cell was very short. ${ }^{10}$

\section{REPRODUCIBILITY OF THE FREEZING TEMPERATURE}

After the acid in each of the five cells had been restored to the condition existing before the series of observations in which the cells were kept at $131.6^{\circ} \mathrm{C}$, a large number of observations were made to determine the reproducibility of the freezing temperature. ${ }^{11}$ Most of these observations were made during a period of 1 month. Four thermometers were used. Two of the thermometers, 535 and 618, were calibrated at the steam point with the high degree of accuracy previously mentioned. This made it possible to assign absolute values to the observed temperatures, although the emphasis in this series of observations was on the constancy and reproducibility of the temperature of a particular cell as measured with a particular thermometer. It should be noted that the observed temperatures have no significance either as the triple point of benzoic acid or as its freezing temperature under 1 atm of dry air, because the conditions in the cells were intermediate between these two defined conditions. The pressure in the cells was about one-third of an atmosphere, but there was some variation in the pressure from cell to cell, and also in the purity of the acid in the several cells, resulting from the way in which the cells were filled.

Figure $3^{12}$ illustrates the course of measurements of a particular cell. The lower curve represents observations made with a thermometer current of $1.00 \mathrm{ma}$ and the upper one with $1.41 \mathrm{ma}$. The average difference between the two sets of measurements, when subtracted from those represented by the lower curve, would give an extrapolated curve of resistances with zero thermometer current. ${ }^{13}$

In table 3 are given six sets of data, all for one cell, but obtained with three thermometers, similar to those used in figure 3 but extrapolated to zero thermometer current.

10 In connection with this discussion, it may be pointed out that the direction and magnitude of the shift in the equilibrium between the acid anhydride and water in the interval between $150^{\circ} \mathrm{C}$ and the freezing point suggest that no measurable amounts of anhydride and water are likely to exist under equilibrium conditions at room temperature.

11 Thanks are expressed to J. L. Clark, of this Bureau, for his help during these observations.

12 The observations in figure 3 were made by the Heat Measurements Section.

13 In accordance with a paper by Mueller (see footnote 7), it has been customary to assume that, in the range from $0^{\circ}$ to $500^{\circ} \mathrm{C}$, the temperature rise produced by a constant thermometer current is independent of the temperature and the medium in which the thermometer is placed. This is true only when the environments of the thermometer do not differ materially with respect to loss of heat at the temperatures being measured. In cases where there is doubt as to whether the heating is the same at all temperatures or in different media at one temperature, the observed resistances may be corrected to those corresponding to zero thermometer current by making observations with two different thermometer currents. For example, the environment of the thermometer in the measurements at the ice point reported in this paper, was shaved ice in water. At the benzoic acid point the thermometer, inserted in the well $B$ of the cell (fig. 1) was separated from the freezing mixture of benzoic acid by the Pyrex thermometer well and a thin air space. Subtracting the difference between the resistances observed when 1.41 and 1.00 ma were used, from the resistance observed when $1.00 \mathrm{ma}$ was used, gave the resistance for zero thermometer current. The difference between the resistances observed for each thermometer, $535,618,515$ and 587 , with thermometer currents of 1.41 and $1.00 \mathrm{ma}$, was $0.000209,0.000184,0.000167$, and $0.000190 \mathrm{ohm}$ at the benzoic acid point. The corresponding differences at the ice point were $0.000133,0.000108,0.000119$, and $0.000120 \mathrm{ohms}$, respectively. The validity of these extrapolations is dependent on the assumption that the heat input and the temperature rise of the thermometers varies as the square of the current, which was verified by making measurements with thermometer currents of $\sqrt{0.50}, 1.00, \sqrt{2.00}$, and $2.00 \mathrm{ma}$. It is interesting to note that if the temperature at the benzoic acid point had been calculated from the resistances observed with 1.00 ma as the thermometer current, the benzoic acid point would have been in error by at least 0.5 millidegree (thermometer 515) and not more than 0.8 millidegree (thermometer 618 ). With a thermometer current of 2 ma, the error would have been 2.0 and 3.2 millidegrees, and larger for higher thermometer currents. 


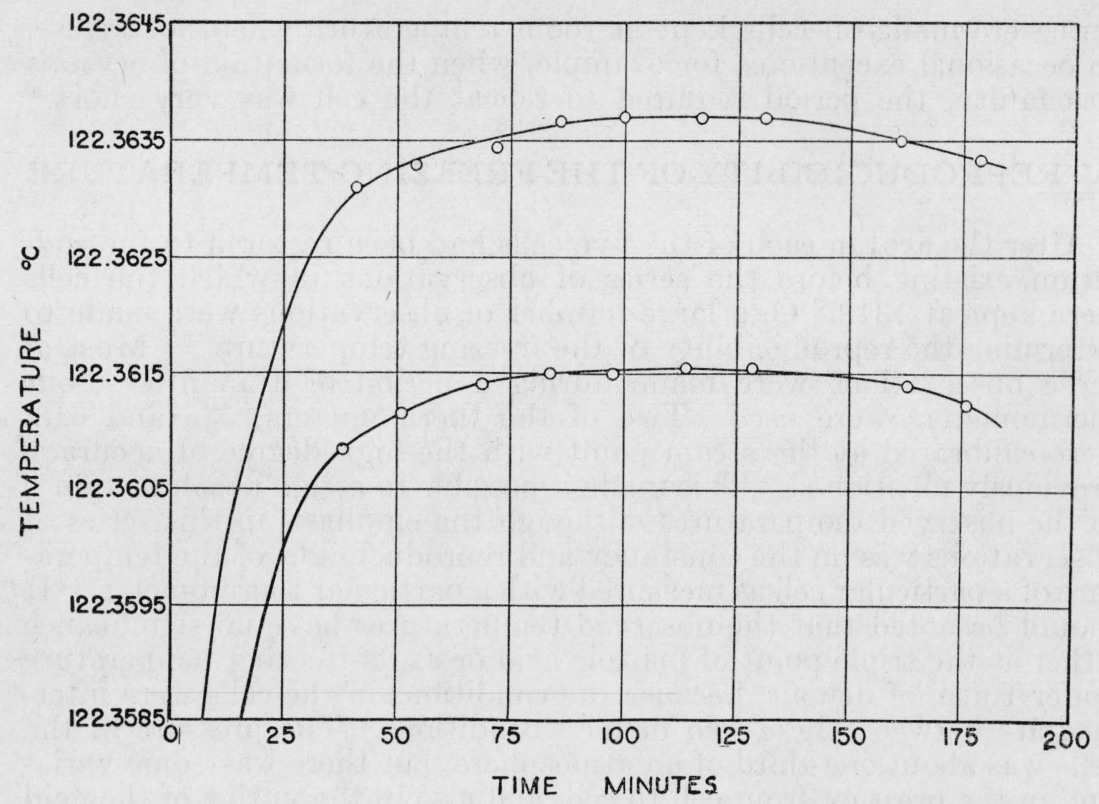

Figure 3.-Constancy of temperature in a benzoic acid fixed-point cell.

Lower curve, Thermometer current $1.00 \mathrm{ma}$; upper curve, thermometer current $1.41 \mathrm{ma}$.

TABLE 3.-Constancy of temperature in a benzoic acid fixed-point cell [Cell 15]

\begin{tabular}{|c|c|c|c|c|c|c|c|c|}
\hline \multicolumn{3}{|c|}{ Thermometer 587} & \multicolumn{3}{|c|}{ Thermometer 535} & \multicolumn{3}{|c|}{ Thermometer 618} \\
\hline Date & Time & $\begin{array}{l}\text { Tempera- } \\
\text { ture }\end{array}$ & Date & Time & $\begin{array}{l}\text { Tempera- } \\
\text { ture }\end{array}$ & Date & Time & $\begin{array}{l}\text { Tempera. } \\
\text { ture }\end{array}$ \\
\hline Aug. 15,1941 & $\left\{\begin{array}{r}\min \\
10 \\
22 \\
34 \\
46 \\
58 \\
82 \\
94 \\
108 \\
118 \\
178\end{array}\right.$ & $\begin{array}{c}{ }^{\circ} \mathrm{C} \\
122.3577 \\
122.3596 \\
122.3600 \\
122.3601 \\
122.3601 \\
122.3600 \\
122.3599 \\
122.3599 \\
122.3599 \\
122.3599\end{array}$ & Aug. 11, 1941 & $\left\{\begin{array}{r}\min \\
12 \\
24 \\
36 \\
46 \\
60 \\
72 \\
84 \\
120 \\
132 \\
180\end{array}\right.$ & $\begin{array}{c}{ }^{\circ} \mathrm{C} \\
122.3602 \\
122.3618 \\
122.3622 \\
122.3624 \\
122.3625 \\
122.3624 \\
122.3627 \\
122.3629 \\
122.3628 \\
122.3626\end{array}$ & Aug. 13, 1941 & $\left\{\begin{array}{r}\min \\
12 \\
24 \\
36 \\
48 \\
60 \\
84 \\
96 \\
108 \\
156\end{array}\right.$ & $\begin{array}{l}{ }^{\circ} C \\
122,3606 \\
122.3623 \\
122.3632 \\
122.3634 \\
122.3634 \\
122.3634 \\
122.3634 \\
122.3635 \\
122.3638\end{array}$ \\
\hline & & & Aug. 12, 1941 & $\left\{\begin{array}{r}11 \\
23 \\
35 \\
47 \\
59 \\
83 \\
95 \\
119\end{array}\right.$ & $\begin{array}{l}122.3605 \\
122.3622 \\
122.3626 \\
122.3627 \\
122.3628 \\
122.3630 \\
122.3630 \\
122.3630\end{array}$ & $\begin{array}{l}\text { Aug. 22, } 1941 \\
\text { Sept. } 29,1941\end{array}$ & $\left\{\begin{array}{r}11 \\
23 \\
35 \\
47 \\
107 \\
\\
6 \\
7 \\
8 \\
9 \\
15 \\
25 \\
38 \\
105 \\
148\end{array}\right.$ & $\begin{array}{l}122.3616 \\
122.3633 \\
122.3637 \\
122.3638 \\
122.3636 \\
122.3625 \\
122.3627 \\
122.3628 \\
122.3629 \\
122.3630 \\
122.3633 \\
122.3636 \\
122.3636 \\
122.3635\end{array}$ \\
\hline
\end{tabular}

- The thermometer was warmed to a temperature near the freezing temperature and then quickly placed in the cell. 
TABLE 4.-Precision of benzoic acid fixed-point cells

\begin{tabular}{|c|c|c|c|c|c|c|c|c|c|c|c|c|c|c|}
\hline \multicolumn{3}{|c|}{ Cell 11} & \multicolumn{3}{|c|}{ Cell 12} & \multicolumn{3}{|c|}{ Cell 13} & \multicolumn{3}{|c|}{ Cell 14} & \multicolumn{3}{|c|}{ Cell 15} \\
\hline Date & 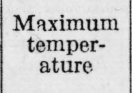 & \begin{tabular}{|c|} 
Variation \\
60 to 120 \\
minutes
\end{tabular} & Date & 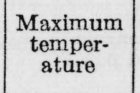 & $\begin{array}{c}\text { Variation } \\
60 \text { to } 120 \\
\text { minutes }\end{array}$ & Date & 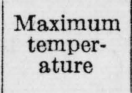 & $\begin{array}{l}\text { Variation } \\
60 \text { to } 120 \\
\text { minutes }\end{array}$ & Date & 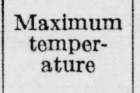 & $\begin{array}{c}\text { Variation } \\
60 \text { to } 120 \\
\text { minutes }\end{array}$ & Date & 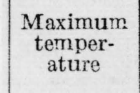 & $\begin{array}{l}\text { Variation } \\
60 \text { to } 120 \\
\text { minutes }\end{array}$ \\
\hline \multicolumn{15}{|c|}{ THERMOMETER 535} \\
\hline 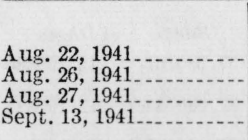 & $\begin{array}{l}{ }^{\circ} \mathrm{C} \\
122.3598 \\
122.3604 \\
122.3608 \\
122.3608 \\
\end{array}$ & \begin{tabular}{c|}
${ }^{\circ} \mathrm{C}$ \\
0.0005 \\
.0005 \\
.0007 \\
.0002
\end{tabular} & 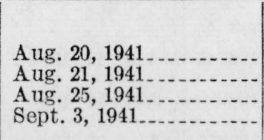 & $\begin{array}{l}{ }^{\circ} \mathrm{C} \\
122.3594 \\
122.3592 \\
122.3593 \\
122.3592 \\
\end{array}$ & $\begin{array}{l}{ }^{\circ} \mathrm{C} \\
0.0007 \\
0.0003 \\
.0005 \\
.0003 \\
\end{array}$ & \multirow{3}{*}{ 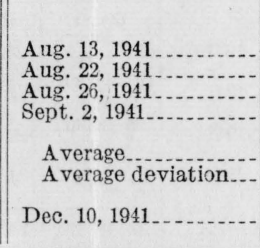 } & \begin{tabular}{|c|}
${ }^{\circ} \mathrm{C}$ \\
122.316 \\
122.3611 \\
122.3611 \\
122.3606 \\
12606
\end{tabular} & \begin{tabular}{c|}
${ }^{\circ} \mathrm{C}$ \\
0.0001 \\
.0005 \\
.0003 \\
.0003
\end{tabular} & \multirow{3}{*}{\begin{tabular}{|l|} 
Aug. 20,1941 \\
Aug. $21,1911 \ldots$ \\
Aug. 27, 1941 \\
Aug. $29,1941 \ldots$ \\
Average \\
Average deviation.... \\
\end{tabular}} & \multirow{3}{*}{\begin{tabular}{|l}
${ }^{\circ} C$ \\
122.3635 \\
122.3635 \\
122.3655 \\
122.3632 \\
$122.3634_{0}$ \\
0.00012
\end{tabular}} & $\begin{array}{l}{ }^{\circ} \mathrm{C} \\
0.0001 \\
.0005 \\
.00002 \\
.0003\end{array}$ & \multirow{3}{*}{ 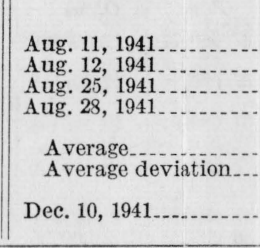 } & \begin{tabular}{c|c|} 
& ${ }^{\circ} \mathrm{C}$ \\
112.3629 \\
\hdashline 122.3630 \\
\hdashline 122.3637 \\
\hdashline 122.3633 \\
\hdashline
\end{tabular} & $\begin{array}{l}{ }^{\circ} \mathrm{C} \\
0.0004 \\
.0002 \\
.00006 \\
.0001\end{array}$ \\
\hline $\begin{array}{l}\text { Average } \\
\text { Average deviation........... }\end{array}$ & $\begin{aligned} 122.335245 \\
0.00035 \\
\end{aligned}$ & $\begin{array}{l}0.00048 \\
0.00014\end{array}$ & $\begin{array}{l}\text { Average } \\
\text { Average deviation........ }\end{array}$ & $\begin{array}{r}122.35928 \\
0.00008 \\
\end{array}$ & $\begin{array}{l}0.0004_{5} \\
0.00015\end{array}$ & & $\begin{array}{r}122.36110 \\
0.00025 \\
\end{array}$ & $\begin{array}{l}0.00030 \\
0.00010\end{array}$ & & & $\begin{array}{l}0.00028 \\
0.00013\end{array}$ & & $\begin{array}{r}122.36325 \\
0.00028 \\
\end{array}$ & $\begin{array}{l}0.00033 \\
0.00018\end{array}$ \\
\hline Dec. 9, 1941_.. & $\mathrm{a}^{\mathrm{a}} 122.3609$ & & Dec. 9, 1941_.. & \begin{tabular}{|l|l|}
$\mathbf{a} 122.3595$ \\
\end{tabular} & 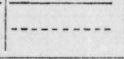 & & $\begin{array}{l}\mathrm{a}_{1} 122.3612 \\
\end{array}$ & & & & & & ${ }^{\mathrm{a}} 122.3631$ & \\
\hline \multicolumn{15}{|c|}{ THERMOMETER 618} \\
\hline 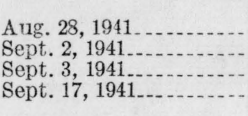 & $\begin{array}{l}122.3609 \\
122.3611 \\
122.3610 \\
122.3612 \\
\end{array}$ & $\begin{array}{r}0.0002 \\
.0003 \\
.0001 \\
.0001 \\
\end{array}$ & \multirow{3}{*}{$\begin{array}{l}\text { Aug. } 26,1941 \\
\text { Aug. } 27,1911 \\
\text { Aug. } 29,1941 \\
\text { Sept. 12, 1941...... } \\
\text { A verage- } \\
\text { Average deviation.... }\end{array}$} & $\begin{array}{l}122.3589 \\
122.3600 \\
122.3594 \\
122.3593 \\
\end{array}$ & $\begin{array}{r}0.0007 \\
0.0073 \\
00003 \\
0.001 \\
\end{array}$ & \multirow{3}{*}{$\begin{array}{l}\text { Aug. 20, 1941 } \\
\text { Aug. } 21,1941 \ldots \\
\text { Aug. 27. 1911 } \\
\text { Aug. 28, 1941 } \\
\text { A verage } \\
\text { A verage deviation.-- }\end{array}$} & $\begin{array}{r}122.3615 \\
122.3617 \\
122.3618 \\
122.3611 \\
\end{array}$ & $\begin{array}{r}0.0003 \\
.0001 \\
.0005 \\
.0001 \\
\end{array}$ & \multirow{3}{*}{$\begin{array}{l}\text { Aug. 12, 1941 } \\
\text { Aug. 25, 1941. } \\
\text { Sept. } 2,1941 \\
\text { Sept. 12, 1941 } \\
\text { Average } \\
\text { Average deviation...- } \\
\end{array}$} & $\begin{array}{l}122.3637 \\
122.3634 \\
122.3627 \\
122.3634 \\
\end{array}$ & $\begin{array}{r}0.0008 \\
.0005 \\
.0003 \\
.0000 \\
\end{array}$ & \multirow{3}{*}{\begin{tabular}{|l} 
Aug. 13, 1941 \\
Aug. 22,1941 \\
Aug. 26, 1941 \\
Aug. 29, $1941 \ldots$ \\
Average- \\
Average deviation \\
Oct. 29, 1941 \\
Dec. 16, 1941_....... \\
\end{tabular}} & $\begin{array}{l}122.3635 \\
122.3638 \\
122.3636 \\
122.3634 \\
\end{array}$ & $\begin{array}{r}0.0001 \\
.0002 \\
.0000 \\
.0002 \\
\end{array}$ \\
\hline $\begin{array}{l}\text { Average } \\
\text { Average deviation.... }\end{array}$ & $\begin{array}{r}122.3610_{5} \\
0.00010 \\
\end{array}$ & $\begin{array}{l}0.00018 \\
0.00008\end{array}$ & & \multirow[t]{2}{*}{$\begin{array}{r}122.3594^{0} \\
0.00030 \\
\end{array}$} & \multirow[t]{2}{*}{$\begin{array}{l}0.0004_{5} \\
0.00025\end{array}$} & & \multirow[t]{2}{*}{$\begin{array}{r}122.3615_{2} \\
0.00026\end{array}$} & \multirow[t]{2}{*}{$\begin{array}{l}0.00025 \\
0.0001_{5}\end{array}$} & & \multirow[t]{2}{*}{$\begin{array}{r}122.36330 \\
0.00030\end{array}$} & \multirow[t]{2}{*}{$\begin{array}{l}0.004_{0} \\
0.00025\end{array}$} & & $\begin{array}{r}122.36358 \\
0.00013 \\
\end{array}$ & $\begin{array}{l}0_{0.00013}^{0} \\
0.00008 \\
\end{array}$ \\
\hline $\begin{array}{l}\text { Sept. } 25,1941 \ldots \\
\text { Oct. } 2,1941 \ldots \ldots\end{array}$ & $\begin{array}{l}\text { a } 122.3613 \\
\text { a } 122.3609 \\
\end{array}$ & $\ldots \ldots$ & & & & & & & & & & & \begin{tabular}{|l|}
$\begin{array}{l}\text { a } 122.3636 \\
a_{122.3630}\end{array}$ \\
\end{tabular} & \\
\hline \multicolumn{15}{|c|}{ THERMOMETER 515} \\
\hline $\begin{array}{l}\text { Aug. } 29,1941 \\
\text { Sept. } 21,1941\end{array}$ & $\begin{array}{l}122.3611 \\
122.3617 \\
\end{array}$ & $\begin{array}{r}0.0004 \\
.0001 \\
\end{array}$ & \multirow{2}{*}{$\begin{array}{l}\text { Aug. 28, 1941. } \\
\text { Sept. 2, 1941...-. } \\
\text { Average- } \\
\text { Average deviation.... }\end{array}$} & $\begin{array}{l}122.3601 \\
122.3610 \\
\end{array}$ & $\begin{array}{r}0.0007 \\
: 0002 \\
\end{array}$ & \multirow{2}{*}{\begin{tabular}{|c|} 
Aug. 29,1941 \\
Sept. 13,1941 \\
A verage- \\
A verage deviation... \\
\end{tabular}} & $\begin{array}{l}122.3617 \\
122.3633 \\
\end{array}$ & $\begin{array}{r}0.0007 \\
.0004\end{array}$ & \multirow{2}{*}{\begin{tabular}{||c|} 
Aup. $28,1941 \ldots$ \\
Sept., $1941 \ldots$ \\
Average \\
Average deviation.....
\end{tabular}} & $\begin{array}{l}122.3640 \\
122.3638 \\
\end{array}$ & $\begin{array}{r}0.0001 \\
.0000 \\
\end{array}$ & \multirow{2}{*}{\begin{tabular}{||c} 
Aug. 27,1941 \\
Sept. 2, 1941_. \\
Average- \\
Average deviation..- \\
Sept. 12, 1941_... \\
\end{tabular}} & $\begin{array}{l}122.3644 \\
122.3637 \\
\end{array}$ & $\begin{array}{r}0.0004 \\
.0002 \\
\end{array}$ \\
\hline $\begin{array}{l}\text { Average- } \\
\text { A verage deviation..... }\end{array}$ & $\begin{array}{l}122.36140 \\
0.00030\end{array}$ & $\begin{array}{l}0.00025 \\
0.00015\end{array}$ & & $\begin{aligned} 122.3605_{5} \\
0.0004_{5}\end{aligned}$ & $\begin{array}{l}0.0004_{5} \\
0.00025\end{array}$ & & $\begin{array}{r}122.36250^{\circ} \\
0.0000_{0}\end{array}$ & $\begin{array}{l}0.0005_{5} \\
0.00011_{5}\end{array}$ & & $\begin{array}{r}122.36390 \\
0.00010^{\circ}\end{array}$ & $\begin{array}{l}0.0000_{5} \\
0.0000_{5}\end{array}$ & & \begin{tabular}{|l|}
$\frac{122.36405}{0.00035}$ \\
${ }^{1} 122.3644$ \\
\end{tabular} & $\begin{array}{l}0.00030 \\
0.00610\end{array}$ \\
\hline \multicolumn{15}{|c|}{ THERMOMETER 587} \\
\hline $\begin{array}{l}\text { Aug. } 20,1941 \\
\text { Aug. } 21,1941 \\
\text { Aug. } 25,1941 \ldots\end{array}$ & $\begin{array}{l}122.3572 \\
122.3571 \\
122.3576 \\
\end{array}$ & $\begin{array}{r}0.0001 \\
.0001 \\
.0002 \\
\end{array}$ & \multirow[t]{2}{*}{ Aug. $22,1941 \ldots$} & \multirow[t]{2}{*}{122.3549} & \multirow[t]{2}{*}{0.0005} & \multirow{2}{*}{\begin{tabular}{|l|} 
Aug. 11,1941 \\
Aug. 12, 1941 \\
Aug. 25, 1911 \\
Average- \\
A verage deviation.... \\
\end{tabular}} & $\begin{array}{l}122.3577 \\
122.3576 \\
122.3582 \\
\end{array}$ & $\begin{array}{r}0.0000 \\
.0002 \\
.0008\end{array}$ & \multirow{2}{*}{\begin{tabular}{|l} 
Aug. 13,1941 \\
Aug. 22,1941 \\
Average \\
Average deviation...-
\end{tabular}} & $\begin{array}{l}122.3599 \\
122.3600 \\
123509 \mathrm{~s}\end{array}$ & $\begin{array}{r}0.0000 \\
.0004 \\
0.0022\end{array}$ & $\begin{array}{l}\text { Aug. 15, } 1941 \\
\text { Aug. 18, } 1941 \\
\text { Aug. } 20,1941 \\
\text { A 21 }\end{array}$ & $\begin{array}{l}122.3601 \\
122.3595 \\
122.3600 \\
112.3600\end{array}$ & $\begin{aligned} 0.0002 \\
.0003 \\
.0003 \\
0.004\end{aligned}$ \\
\hline $\begin{array}{l}\text { Average } \\
\text { Average deviation...- }\end{array}$ & $\begin{array}{r}122.35733_{0} \\
0.00220\end{array}$ & $\begin{array}{l}0.00013 \\
0.00004\end{array}$ & & & & & $\begin{array}{r}122.35788_{3} \\
0.00024\end{array}$ & $\begin{array}{l}0.00033 \\
0.00030\end{array}$ & & $\begin{array}{r}122.3599_{5} \\
0.000_{5}\end{array}$ & $\begin{array}{l}0.00020 \\
0.00020\end{array}$ & $\begin{array}{l}\text { Aug. } 21,1941 \\
\text { Average } \\
\text { Average deviation-. } \\
\text { Dec. } 18,1941\end{array}$ & \begin{tabular}{|c|}
$\frac{122.3600}{122.35990}$ \\
0.00020 \\
${ }_{1} 122.3634$
\end{tabular} & $\begin{array}{l}.00004 \\
0.00030 \\
0.0000_{8}\end{array}$ \\
\hline
\end{tabular}

- Values not included in the averages.

634436-45 (Face p. 346) 
TABLE 5.-Measurements of the ice and benzoic acid points

\begin{tabular}{|c|c|c|c|c|c|c|c|c|c|c|c|c|c|c|c|c|c|c|c|c|c|c|c|c|}
\hline \multicolumn{5}{|c|}{ Cell 11} & \multicolumn{5}{|c|}{ Cell 12} & \multicolumn{5}{|c|}{ Cell 13} & \multicolumn{5}{|c|}{ Cell 14} & \multicolumn{5}{|c|}{ Cell 15} \\
\hline Date & Ice point & $\begin{array}{c}\text { Average } \\
\text { ice point }\end{array}$ & $\begin{array}{c}\text { Benzoic } \\
\text { acid point }\end{array}$ & $\Delta R$ & Date & Ice point & $\begin{array}{c}\text { Average } \\
\text { ice point }\end{array}$ & $\begin{array}{l}\text { Benzoic } \\
\text { acid point }\end{array}$ & $\Delta R$ & Date & Ice point & $\begin{array}{c}\text { Average } \\
\text { ice point }\end{array}$ & $\mid \begin{array}{c}\text { Benzoic } \\
\text { acid point }\end{array}$ & $\Delta R$ & Date & Ice point & $\begin{array}{c}\text { Average } \\
\text { ice point }\end{array}$ & $\begin{array}{c}\text { Benzoic } \\
\text { acid point }\end{array}$ & $\Delta R$ & Date & Ice point & $\begin{array}{c}\text { A verage } \\
\text { ice point }\end{array}$ & \begin{tabular}{|c|} 
Benzoic \\
acid point
\end{tabular} \mid & $\Delta R$ \\
\hline \multicolumn{5}{|c|}{ THERMOMETER 535} & \multicolumn{5}{|c|}{ THERMOMETER 535} & \multicolumn{5}{|c|}{ THERMOMETER 535} & \multicolumn{5}{|c|}{ THERMOMETER 535} & \multicolumn{5}{|c|}{ THERMOMETER 535} \\
\hline $\begin{array}{l}\text { Aug. } 22,1941 \ldots \\
\text { Aug. } 26,1941 \ldots \\
\text { Aug. } 27,1941 \ldots \\
\text { Sept. } 13,1941\end{array}$ & 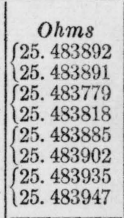 & $\mid \begin{array}{c}\text { Ohms } \\
25.488891 \\
\{25.483799 \\
25.488894 \\
255.483941\end{array}$ & \begin{tabular}{|c|} 
Ohms \\
37.680482 \\
37.680400 \\
37.680581 \\
37.680046
\end{tabular} & \begin{tabular}{c|} 
Ohms \\
12. 196591 \\
12. 196601 \\
12. 196687 \\
12. 196705
\end{tabular} & $\begin{array}{l}\text { Aug. } 20,1941 \\
\text { Aug. } 21,1941 \\
\text { Aug. } 25,1941 \\
\text { Sept. } 3,1941\end{array}$ & 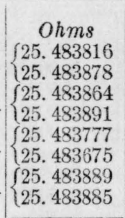 & $\left\{\begin{array}{c}\text { Ohms } \\
225.483847 \\
25.483878 \\
25.483726 \\
255.483887\end{array}\right.$ & \begin{tabular}{|c|} 
Ohms \\
37.680377 \\
37.683996 \\
37.680191 \\
37.680418
\end{tabular} & $\begin{array}{l}\text { Ohms } \\
\text { 12. } 196530 \\
\text { 12. } 196518 \\
\text { 12. } 196465 \\
\text { 12. } 196531\end{array}$ & \begin{tabular}{|l|} 
Aug. $13,1941 \ldots$ \\
Aug. $22,1941 \ldots$ \\
Aug. $26,1941 \ldots$ \\
Sept. 2, 1941 \\
\end{tabular} & 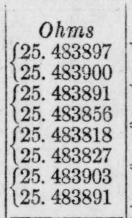 & $\mid$\begin{tabular}{r|} 
Ohms \\
25.483899 \\
25.483874 \\
25.483823 \\
25.483897
\end{tabular} & \begin{tabular}{|c|} 
Ohms \\
37.680662 \\
37.680581 \\
37.680502 \\
37.680570
\end{tabular} & $\begin{array}{l}\text { Ohms } \\
12.196763 \\
12.196707 \\
12.196679 \\
12.196672\end{array}$ & \begin{tabular}{|l} 
Aug. $20,1941 \ldots$ \\
Aug. $21,1941 \ldots$ \\
Aug. $27,1941 \ldots$ \\
Sept. $29,1941 \ldots$
\end{tabular} & 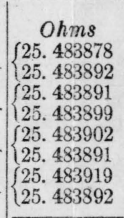 & $\mid \begin{array}{c}\text { Ohms } \\
225.483885 \\
25.483895 \\
25.483897 \\
255.483906\end{array}$ & \begin{tabular}{|c|} 
Ohms \\
37.680828 \\
37.680845 \\
37.680348 \\
37.680833
\end{tabular} & \begin{tabular}{l||} 
Ohms \\
12. 196943 \\
12. 196950 \\
12. 196951 \\
12. 196927
\end{tabular} & 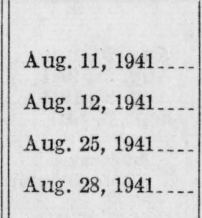 & 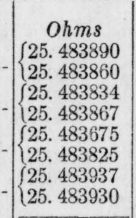 & $\left|\begin{array}{c}\text { Ohms } \\
225.483875 \\
25.483851 \\
25.483750 \\
255.483934\end{array}\right|$ & \begin{tabular}{|c|} 
Ohms \\
37.680761 \\
37.680735 \\
37.680657 \\
37.680889
\end{tabular} & $\begin{array}{l}\text { Ohms } \\
12.196886 \\
12.196884 \\
12.196907 \\
12.196955\end{array}$ \\
\hline $\begin{array}{l}\text { Average } \\
\text { Rangea } \\
\text { Average devias } \\
\text { tion. }\end{array}$ & $\begin{array}{r}25.483881 \\
0.000168 \\
0.000041\end{array}$ & $\begin{array}{r}25.483881 \\
0.000142 \\
0.000036\end{array}$ & $\begin{array}{r}37.680527 \\
0.000246 \\
0.000086\end{array}$ & $\begin{array}{r}12.196646 \\
0.000114 \\
0.000050\end{array}$ & $\begin{array}{l}\text { Average-. } \\
\text { Rangag } \\
\text { Average devia- } \\
\text { tion. }\end{array}$ & $\begin{array}{r}25.483835 \\
0.000216 \\
0.000059\end{array}$ & $\begin{array}{r}25.483835 \\
0.000161 \\
0.000054\end{array}$ & $\begin{array}{r}37.680346 \\
0.000227 \\
0.000077\end{array}$ & $\begin{array}{l}12.196511 \\
0.000066 \\
0.000023\end{array}$ & $\begin{array}{l}\text { Average... } \\
\text { Rangea } \\
\text { Average devia- } \\
\text { tion. }\end{array}$ & $\begin{array}{r}25.483873 \\
0.000035 \\
0.000029 \\
\end{array}$ & $\begin{array}{r}25.483873 \\
0.000076 \\
0.000025\end{array}$ & $\begin{array}{r}37.680579 \\
0.0000160 \\
0.000043\end{array}$ & $\begin{array}{r}12.196706 \\
0.000091 \\
0.000030\end{array}$ & \begin{tabular}{|l} 
Average. \\
Rangeg. \\
A verage devia- \\
tion.
\end{tabular} & $\begin{array}{r}25.483896 \\
0.000041 \\
0.000009\end{array}$ & $\begin{array}{r}25.483896 \\
0.000021 \\
0.000006\end{array}$ & $\begin{array}{r}37.680839 \\
0.000020 \\
0.000000\end{array}$ & $\begin{array}{r}12.196943 \\
0.000024 \\
0.000008\end{array}$ & \begin{tabular}{|l} 
A verage \\
Rangeg \\
A verage devia- \\
tion.
\end{tabular} & $\begin{array}{r}25.483852 \\
0.000222 \\
0.000056\end{array}$ & $\begin{array}{r}25.483852 \\
0.000184 \\
0.000052\end{array}$ & \begin{tabular}{|}
37.680761 \\
0.000232 \\
0.000040
\end{tabular} & $\begin{array}{r}12.196908 \\
0.000071 \\
0.000026\end{array}$ \\
\hline \multicolumn{5}{|c|}{ THERMOMETER 618} & \multicolumn{5}{|c|}{ THERMOMETER 618} & \multicolumn{5}{|c|}{ THERMOMETER 618} & \multicolumn{5}{|c|}{ THERMOMETER 618} & \multicolumn{5}{|c|}{ THERMOMETER 618} \\
\hline $\begin{array}{l}\text { Aug. 28, 1941_. } \\
\text { Sept. } 2,1941 \ldots \\
\text { Sept. 3, 1941_... } \\
\text { Sept. 17, 1941_... }\end{array}$ & 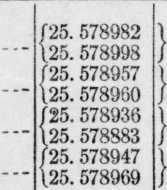 & $\left\{\begin{array}{l}25.578990 \\
25.578959 \\
25.578910 \\
25.578958\end{array}\right.$ & $\begin{array}{l}37.819871 \\
37.819849 \\
37.819765 \\
37.819855 \\
\end{array}$ & \begin{tabular}{l|}
12.210881 \\
12.240890 \\
12.240855 \\
12.240897
\end{tabular} & $\begin{array}{l}\text { Aug. } 26,1941 \ldots \\
\text { Aug. } 27,1941 \ldots \\
\text { Aug. } 29,1941 \ldots \\
\text { Sept. } 12,1941 \ldots\end{array}$ & 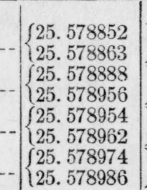 & $\left\{\begin{array}{l}25.578858 \\
25.578922 \\
25.578958 \\
25.578980\end{array}\right.$ & \begin{tabular}{|l|}
37.819437 \\
37.819682 \\
37.819675 \\
37.819699 \\
\end{tabular} & \begin{tabular}{|l|}
12.240629 \\
12.240760 \\
12.240717 \\
12.240719
\end{tabular} & \begin{tabular}{|l|} 
Aug. $20,1941 \ldots$ \\
Aug. $21,1941 \ldots$ \\
Aug. 27, $1941 \ldots$ \\
Aug. 28, 1941 $\ldots$ \\
\end{tabular} & 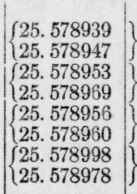 & $\left\{\begin{array}{l}25.578943 \\
25.578961 \\
25.578958 \\
25.578988\end{array}\right.$ & \begin{tabular}{|l|}
37.819862 \\
37.819910 \\
37.819912 \\
37.819890
\end{tabular} & \begin{tabular}{r|}
12.240919 \\
12.240949 \\
12.240954 \\
12.240902
\end{tabular} & 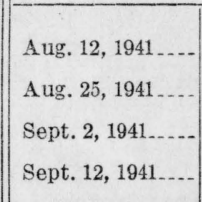 & 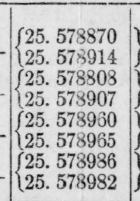 & $\left\{\begin{array}{l}25.578892 \\
25.578858 \\
25.578963 \\
25.578984\end{array}\right.$ & $\begin{array}{l}37.820009 \\
37.819922 \\
37.820009 \\
37.820110 \\
\end{array}$ & \begin{tabular}{l||}
12.241117 \\
12.241064 \\
12.241046 \\
12.241126
\end{tabular} & 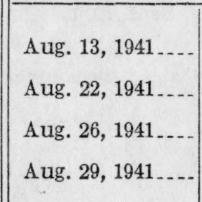 & $\left\{\begin{array}{l}25.575018 \\
25.579015 \\
25.578954 \\
225.578911 \\
25.578683 \\
25.578894 \\
25.578962 \\
25.579023\end{array}\right\}$ & $\left\{\begin{array}{l}25.579017 \\
25.578933 \\
25.578879 \\
25.578993\end{array}\right.$ & \begin{tabular}{|l|}
37.820166 \\
37.820070 \\
37.819979 \\
37.820122
\end{tabular} & $\begin{array}{l}12.241149 \\
12.241137 \\
12.241100 \\
12.241129\end{array}$ \\
\hline $\begin{array}{l}\text { Average } \\
\text { Range } \\
\text { Average devia- } \\
\text { tion. }\end{array}$ & $\begin{array}{r}25.578954 \\
0.000115 \\
0.000024\end{array}$ & \begin{tabular}{r|}
25.578954 \\
0.000080 \\
0.000022
\end{tabular} & $\begin{array}{r}37.819835 \\
0.000105 \\
0.000025\end{array}$ & $\begin{array}{r}12.240881 \\
0.000042 \\
0.0000013\end{array}$ & $\begin{array}{l}\text { Average- } \\
\text { Range } \\
\text { Average devia- } \\
\text { tion. }\end{array}$ & $\begin{array}{c}25.578929 \\
0.000134 \\
0.000046\end{array}$ & $\begin{array}{r}25.578930 \\
0.000122 \\
0.000040\end{array}$ & $\begin{array}{r}37.819636 \\
0.000212 \\
0.000074\end{array}$ & $\begin{array}{r}12.2440706 \\
0.000131 \\
0.000039\end{array}$ & $\begin{array}{l}\text { Average. } \\
\text { Range } \\
\text { A verage devia- } \\
\text { tion. }\end{array}$ & \begin{tabular}{|r|}
25.578963 \\
0.000059 \\
0.000015
\end{tabular} & $\begin{array}{r}25.578963 \\
0.000045 \\
0.000013\end{array}$ & \begin{tabular}{r|}
37.819894 \\
0.000050 \\
0.000018
\end{tabular} & $\begin{array}{r}12.240931 \\
0.000052 \\
0.000021\end{array}$ & \begin{tabular}{|l} 
Average \\
Range... \\
Average devia- \\
tion.
\end{tabular} & $\begin{array}{r}25.578924 \\
0.000178 \\
0.000000\end{array}$ & \begin{tabular}{|r|}
25.578924 \\
0.000126 \\
0.000049
\end{tabular} & $\begin{array}{r}37.820012 \\
0.000188 \\
0.000049\end{array}$ & \begin{tabular}{r|}
12.2410838 \\
0.000080 \\
0.000033
\end{tabular} & \begin{tabular}{|l} 
Averaga \\
Range...... \\
Average devia- \\
tion.
\end{tabular} & $\mid \begin{array}{r}25.578956 \\
0.000160 \\
0.000050\end{array}$ & $\begin{array}{r}25.578955 \\
0.000138 \\
0.000050\end{array}$ & $\begin{array}{r}37.820084 \\
0.000187 \\
0.000060\end{array}$ & $\begin{array}{r}12.241129 \\
0.00009 \\
0.000014\end{array}$ \\
\hline \multicolumn{5}{|c|}{ THERMOMETER 515} & \multicolumn{5}{|c|}{ THERMOMETER 515} & \multicolumn{5}{|c|}{ THERMOMETER 515} & \multicolumn{5}{|c|}{ THERMOMETER 515} & \multicolumn{5}{|c|}{ THERMOMETER 515} \\
\hline $\begin{array}{l}\text { Aug. 29, } 1941 \ldots \\
\text { Sept. 12, } 1941 \ldots\end{array}$ & \multirow{2}{*}{$\begin{array}{c}25.4832929 \\
25.4832525 \\
255.438326 \\
25.483316\end{array}$} & \multirow{2}{*}{$\left|\begin{array}{r}25.483280 \\
25.483321 \\
25.483301 \\
0.000041 \\
0.000021\end{array}\right|$} & $\begin{array}{l}37.673587 \\
37.673701\end{array}$ & $\begin{array}{l}12.190307 \\
12.190380\end{array}$ & $\begin{array}{l}\text { Aug. } 28,1941 \ldots \\
\text { Sept. 2, } 1941 \ldots\end{array}$ & $\left\{\begin{array}{l}25.483300 \\
25.433332 \\
25.43859 \\
25.433216\end{array}\right.$ & $\left\{\begin{array}{l}25.483316 \\
25.483238\end{array}\right.$ & $\begin{array}{l}37.673537 \\
37.673530 \\
\end{array}$ & $\begin{array}{l}12.190221 \\
12.190292\end{array}$ & $\mid$\begin{tabular}{|l} 
Aug. $29,1941 \ldots$ \\
Sept. $13,1941 \ldots$
\end{tabular} & $\left\{\begin{array}{l}25.4832255 \\
25.4832777 \\
25.483235 \\
25.483243\end{array} \mid\right.$ & $\left\{\begin{array}{l}25.483271 \\
25.483239\end{array}\right.$ & \begin{tabular}{|l|}
37.673629 \\
37.673739 \\
\end{tabular} & $\begin{array}{l}12.190358 \\
12.190500 \\
\end{array}$ & \begin{tabular}{|l} 
Aug. $28,1941 \ldots$ \\
Sept. $9,1941 \ldots$
\end{tabular} & $\left\{\begin{array}{l}25.483332 \\
25.483292 \\
25.483260 \\
25.438267\end{array}\right.$ & $\left\{\begin{array}{l}25.483312 \\
25.483264\end{array}\right.$ & $\begin{array}{l}37.673911 \\
37.673825 \\
\end{array}$ & \begin{tabular}{l|l|} 
12. 190599 \\
12. 190561
\end{tabular} & \begin{tabular}{|l} 
Aug. $27,1941 \ldots \ldots$ \\
Sept. 2, 1941_....
\end{tabular} & $\left\{\begin{array}{l}\{5.483278 \\
25.438398 \\
25.438261 \\
25.483279\end{array}\right\}$ & $\left\{\begin{array}{l}25.483288 \\
25.483270\end{array}\right.$ & \begin{tabular}{|l|}
37.673923 \\
37.678825 \\
\end{tabular} & $\begin{array}{r}12.190635 \\
12.190555 \\
\end{array}$ \\
\hline $\begin{array}{l}\text { Average- } \\
\begin{array}{l}\text { Range } \\
\text { Average devia- } \\
\text { tion. }\end{array}\end{array}$ & & & $\begin{array}{r}37.673564 \\
0.000014 \\
0.000057\end{array}$ & $\begin{array}{r}12.190344 \\
0.000073 \\
0.000036\end{array}$ & $\begin{array}{l}\text { Average-_. } \\
\text { Range } \\
\text { Average devia- } \\
\text { tion. }\end{array}$ & $\begin{array}{r}25.483277 \\
0.00016 \\
0.000039\end{array}$ & $\begin{array}{r}25.483277 \\
0.000078 \\
0.000039\end{array}$ & $\begin{array}{r}37.673534 \\
0.000007 \\
0.000004\end{array}$ & $\begin{array}{r}12.190257 \\
0.000071 \\
0.000036\end{array}$ & \begin{tabular}{|l} 
Average \\
Range. \\
A verage devia- \\
tion.
\end{tabular} & \begin{tabular}{|r|}
25.483255 \\
0.000042 \\
0.000016
\end{tabular} & $\begin{array}{r}25.483255 \\
0.000032 \\
0.000016\end{array}$ & $\begin{array}{r}37.673864 \\
0.000110 \\
0.000055\end{array}$ & $\begin{array}{r}12.190429 \\
0.000142 \\
0.000071\end{array}$ & $\begin{array}{l}\text { Average } \\
\text { Range...- } \\
\text { A verage devia- } \\
\text { tion. }\end{array}$ & $\begin{array}{c}25.483288 \\
0.00072 \\
0.000024\end{array}$ & $\begin{array}{r}25.483288 \\
0.0000048 \\
0.000024\end{array}$ & $\begin{array}{r}37.673868 \\
0.000036 \\
0.000043\end{array}$ & $\begin{array}{r}12.190580 \\
0.000038 \\
0.000019\end{array}$ & \begin{tabular}{||l} 
Average \\
Range...- \\
Average devia- \\
tion.
\end{tabular} & $\begin{array}{r}25.483279 \\
0.000037 \\
0.000010\end{array}$ & $\begin{array}{r}25.483279 \\
0.000018 \\
0.000009\end{array}$ & $\begin{array}{r}37.673874 \\
0.000098 \\
0.000049\end{array}$ & $\begin{array}{r}12.190595 \\
0.000030 \\
0.000040\end{array}$ \\
\hline & THERM & IOMETER & 587 & & & THERM & MOMETER & 587 & & & THERM & TOMETER & 587 & & & THERM & MOMETER & & & & THERM & ER & & \\
\hline $\begin{array}{l}\text { Aug. 20, } 1941 \ldots \\
\text { Aug. } 21,1941 \ldots \\
\text { Aug. } 25,1941 \ldots\end{array}$ & 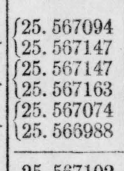 & $\left\{\begin{array}{l}25.567121 \\
25.567155 \\
25.567031 \\
\end{array}\right.$ & $\begin{array}{l}37.803281 \\
37.803319 \\
37.803160 \\
\end{array}$ & \begin{tabular}{r|}
12.236150 \\
12.236164 \\
12.236144 \\
\end{tabular} & Aug. $22,1941 \ldots$ & $\left\{\begin{array}{l}25.567151 \\
225.567144\end{array}\right.$ & 25.587150 & 37.803100 & 12. 235950 & \begin{tabular}{|l} 
Aug. 11, $1941 \ldots$ \\
Aug. 12, 1941_... \\
Aug. 25, 1941 $\ldots$
\end{tabular} & 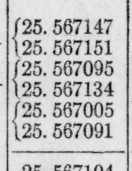 & $\left\{\begin{array}{l}25.567149 \\
25.567115 \\
25.567048\end{array}\right.$ & \begin{tabular}{|l|}
37.803374 \\
37.803311 \\
37.803274 \\
27.00230 \\
\end{tabular} & $\begin{array}{r}12.236225 \\
12.236196 \\
12.236226 \\
\end{array}$ & \begin{tabular}{|l} 
Aug. 13, $1941 \ldots . .$. \\
Aug. 22, 1941_... \\
Average_......... \\
Range...
\end{tabular} & 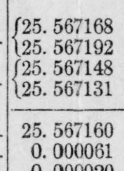 & $\left|\begin{array}{r}25.567180 \\
25.567140 \\
25.567160 \\
0.000000\end{array}\right|$ & \begin{tabular}{|r|}
37.803630 \\
37.803578 \\
37.803604 \\
0.000052
\end{tabular} & \begin{tabular}{r||}
12.236450 \\
12.236438 \\
12.236444 \\
0.000012
\end{tabular} & 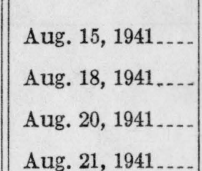 & 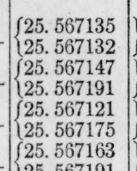 & $\left\{\begin{array}{l}25.567134 \\
25.567169 \\
25.567148 \\
25.567177\end{array} \mid\right.$ & \begin{tabular}{|l|}
37.803587 \\
37.803572 \\
37.803594 \\
37.803642
\end{tabular} & $\begin{array}{l}12.236434 \\
12.236403 \\
12.236446 \\
12.236465\end{array}$ \\
\hline $\begin{array}{l}\text { Average } \\
\text { Ranga } \\
\text { Average devia- } \\
\text { tion. }\end{array}$ & $\begin{array}{r}25.567102 \\
0.000175 \\
0.000050\end{array}$ & \begin{tabular}{r|}
25.567102 \\
0.000124 \\
0.000048
\end{tabular} & $\begin{array}{r}37.803253 \\
0.000159 \\
0.000062\end{array}$ & $\begin{array}{r}12.236156 \\
0.000020 \\
0.000000\end{array}$ & & & & & & $\begin{array}{l}\text { Average } \\
\text { Range..... } \\
\text { Average devia- } \\
\text { tion. }\end{array}$ & \begin{tabular}{|r|}
25.567104 \\
0.000146 \\
0.000040
\end{tabular} & \begin{tabular}{|r|}
25.567104 \\
0.0000101 \\
0.000037
\end{tabular} & $\begin{array}{c}37.803232 \\
0.000000 \\
0.000035\end{array}$ & $\begin{array}{r}12.236216 \\
0.000030 \\
0.000013\end{array}$ & 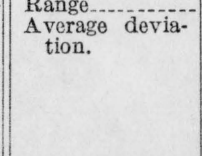 & $\begin{array}{l}0.000001 \\
0.00020\end{array}$ & 0.000040 & $\begin{array}{l}0.0000022 \\
0.00025\end{array}$ & $\begin{array}{l}0.0000012 \\
0.00006\end{array}$ & \begin{tabular}{||l|} 
Aug. 21, 1941_.... \\
A Average....... \\
Rangea. \\
Average devia- \\
tion.
\end{tabular} & $\mid \begin{array}{r}25.556767191 \\
\begin{array}{r}25.56767177 \\
0.000070 \\
0.000024\end{array}\end{array}$ & $\left|\begin{array}{|c|}25.567177 \\
25.567157 \\
0.000043 \\
0.000016\end{array}\right|$ & $\left|\begin{array}{r|}37.803642 \\
37.803599 \\
0.0000070 \\
0.000025\end{array}\right|$ & $\begin{array}{r}12.236465 \\
\begin{array}{r}12.236437 \\
0.000032 \\
0.000019\end{array}\end{array}$ \\
\hline
\end{tabular}


In table 4 are recorded the systematic observations of five cells with the four thermometers. In addition to the date of each measurement, there are given the maximum observed temperature and the variation during the time interval 60 to 120 minutes. Also given are the averages of each set of measurements with a particular cell and thermometer and the average deviation of these measurements. Following these averages are single measurements of each of four cells with thermometer 535, and measurements of one cell (15) with each of the four thermometers, all made about 3 months after the others. At that time (Dec. 18, 1941, table 4) thermometer 587 had also been calibrated at the steam point with the same accuracy as 535 and 618 , hence the measurements made with the three thermometers are directly comparable.

For a few days toward the end of the period during which the systematic observations were made, the humidity became so high that it had a significant effect on the behavior of the bridge and galvanometer. This was apparent from erratic observations of the ice point. Only those measurements that were unmistakably unreliable from this cause have been excluded from table 4 .

\section{RELATIVE REPRODUCIBILITY OF THE ICE POINT AND THE BENZOIC ACID POINT}

In table 5 the resistances observed at the ice point and at the benzoic acid point have been listed to show the relative reproducibilities of these two fixed points. The average of the average deviations of the difference of resistances between the ice and benzoic acid points is $0.000025_{7} \mathrm{ohm}$. The greatest difference between any two observations of the resistance, in a set of observations with one thermometer, was $0.000262 \mathrm{ohm}$ at the ice point (cell 15, thermometer 535 ) and $0.000246 \mathrm{ohm}$ at the benzoic acid point (cell 11, thermometer 535).

The average of the average deviations obtained from the respective averages of each set of observations is $0.000032_{8} \mathrm{ohm}$ at the ice point and $0.000043_{9} \mathrm{ohm}$ at the benzoic acid point. These two averages are in about the same ratio to each other as that of the respective total resistances, 25 to $37 \mathrm{ohms}$. This suggests that the main source of variability in the observations, both of the ice point and of the benzoic acid point, may have been in the bridge. If this was true, both fixed points were reproduced with about equal precision, and possibly with a greater actual precision than is shown by the recorded resistances.

\section{COMPARISON OF THERMOMETERS}

In table 6 the average maximum temperatures observed in each cell with each thermometer have been combined to show the differences between observations of a particular cell with all possible pairs of the four thermometers. It will be seen that the differences between thermometers 535 and 618 are the smallest. As previously noted, these thermometers had received the specially accurate calibration at the steam point. The other two were calibrated in accordance with the routine practice of the Thermometry Section. In view of the reproducibility of the freezing temperature of each cell, as shown by table 4, it may reasonably be concluded that the comparisons of all other 
pairs of thermometers reflect uncertainties in the calibration of resistance thermometers as normally practiced. In view of the known reproducibility of the ice point, nearly all of the uncertainty must result from observations of the steam point. Assuming no error in calibration at the ice point, the interpolated error at the steam point is four-fifths the error at the benzoic acid point. These uncertainties are no larger than can be expected from the errors of barometric measurements. The largest difference, that between thermometers 515 and 587 , corresponds to a difference of $0.09 \mathrm{~mm}$ in the respective barometric readings.

TABLE 6.-Comparison of thermometers a

\begin{tabular}{|c|c|c|c|c|c|c|}
\hline Cell & $T_{535}-T_{587}$ & $T_{618}-T_{587}$ & $T_{618}-T_{535}$ & $T_{515}-T_{587}$ & $T_{515}-T_{535}$ & $T_{515}-T_{618}$ \\
\hline $\begin{array}{l}11 \\
12 \\
13 \\
15\end{array}$ & $\begin{array}{l}{ }^{\circ} \mathrm{C} \\
0.0031_{5} \\
.0043_{8} \\
.0032_{7} \\
.0034_{5} \\
.0033_{5}\end{array}$ & $\begin{array}{l}{ }^{\circ} \mathrm{C} \\
0.00375 \\
.0045_{0} \\
.0036_{9} \\
.0033_{5} \\
.0036_{8}\end{array}$ & $\begin{array}{l}{ }^{\circ} C \\
0.0006_{0} \\
.0001_{2} \\
.0004_{2} \\
.0001_{0} \\
.0003_{3}\end{array}$ & $\begin{array}{l}{ }^{\circ} \mathrm{C} \\
0.041_{0} \\
.0056_{5} \\
.0046_{7} \\
.0039_{5} \\
.0041_{5}\end{array}$ & $\begin{array}{l}{ }^{\circ} C \\
0.0009_{5} \\
.0012_{7} \\
.0014_{0} \\
.00050 \\
.0008_{0}\end{array}$ & $\begin{array}{l}{ }^{\circ} \mathrm{C} \\
0.0003_{5} \\
.0011_{2} \\
.0009_{8} \\
.0006_{0} \\
.0004_{7}\end{array}$ \\
\hline $\begin{array}{l}\text { Average } \\
\text { A verage deviation }\end{array}$ & $\begin{array}{l}0.0035_{2} \\
0.0003_{4}\end{array}$ & $\begin{array}{l}0.00379 \\
0.00028\end{array}$ & $\begin{array}{l}0.0002_{7} \\
0.0002_{1}\end{array}$ & $\begin{array}{l}0.0045_{0} \\
0.0005_{9}\end{array}$ & $\begin{array}{l}0.0009_{8} \\
0.0003_{5}\end{array}$ & $\begin{array}{l}0.0007_{1} \\
0.0003_{6}\end{array}$ \\
\hline
\end{tabular}

a $T$ is the average maximum temperature of a set of observations as given under each of the cells in table 4. The subscripts to $T$ define the thermometers with which the measurements were made.

\section{COMPARISON OF CELLS}

In table 7 the data of table 4 are combined so as to show the differences between the maximum temperatures of all possible pairs of cells as measured with each of the four thermometers. The greatest difference between any pair (cells 12 and 15) is 4.2 millidegrees, and the smallest (between cells 14 and 15 ) is 0.05 millidegree. As previously noted, these differences are to be attributed partly to differences in the amount of benzoic anhydride or of water contained in the acid as the result of variations in the conditions under which the cells were filled. The differences may also result in part from differences in pressure of atmospheric gases in the cells when freezing begins. Although the pressures were nearly the same in all of the cells when they were sealed at room temperature, the pressures differed at the freezing point because the volume of free space (vapor space) was not closely controlled and the expansion which occurred as the cells were heated to the freezing temperature of the acid caused relative differences in the volumes of the free space. These differences between the freezing temperatures in different cells can undoubtedly be made smaller by more careful control of the indicated factors. The freezing temperatures can also be brought very close to a defined temperature, most readily to the triple point, by proper attention to the factors of purity and of pressure existing within the cell when freezing begins. 
TABLE 7.-Comparison of cells a

\begin{tabular}{|c|c|c|c|c|c|c|c|c|c|c|}
\hline Thermometer & $T_{12}-T_{11}$ & $T_{13}-T_{11}$ & $T_{14}-T_{11}$ & $T_{15}-T_{11}$ & $T_{13}-T_{12}$ & $T_{14}-T_{12}$ & $T_{15}-T_{12}$ & $T_{14}-T_{13}$ & $T_{15}-T_{13}$ & $T_{15}-T_{14}$ \\
\hline - & $\begin{array}{c}{ }^{\circ} C \\
-0.0011_{7} \\
-.0016_{5} \\
-.0008_{5} \\
-.0024_{0}\end{array}$ & $\begin{array}{c}\circ C \\
0.00065 \\
.00047 \\
.00110 \\
.00053\end{array}$ & $\begin{array}{c}\circ C \\
0.0029_{5} \\
.0022_{5} \\
.0025_{0} \\
.0026_{5}\end{array}$ & $\begin{array}{c}\circ C \\
0.0028_{0} \\
.00253 \\
.0026 \\
.00260\end{array}$ & \begin{tabular}{c}
$\circ C$ \\
$0.0018_{2}$ \\
$.0021_{2}$ \\
$.0019_{5}$ \\
\hdashline--
\end{tabular} & $\begin{array}{c}{ }^{\circ} C \\
0.0041_{2} \\
.00390 \\
.00335 \\
-\end{array}$ & $\begin{array}{c}\circ C \\
0.0039_{7} \\
.0041_{8} \\
.00350 \\
-\cdot-\end{array}$ & $\begin{array}{c}\circ C \\
0.0023_{0} \\
.0017 \\
.00140 \\
.0021_{2}\end{array}$ & $\begin{array}{c}\circ C \\
0.0021_{5} \\
.0020_{6} \\
.0015 \\
.00207\end{array}$ & $\begin{array}{r}\circ C \\
-0.00015 \\
.00028 \\
.00015 \\
-.00005\end{array}$ \\
\hline $\begin{array}{l}\text { A verage } \\
\text { A verage Devia- } \\
\text { tion. }\end{array}$ & $\begin{array}{r}-0.0012_{2} \\
0.0002_{8}\end{array}$ & $\begin{array}{l}0.0006_{9} \\
0.0002_{1}\end{array}$ & $\begin{array}{l}0.0025_{7} \\
0.0002_{1}\end{array}$ & $\begin{array}{l}0.0026_{5} \\
0.0000_{8}\end{array}$ & $\begin{array}{l}0.0019_{6} \\
0.0001_{0}\end{array}$ & $\begin{array}{l}0.0037_{9} \\
0.0002_{9}\end{array}$ & $\begin{array}{l}0.0038_{8} \\
0.0002_{6}\end{array}$ & $\begin{array}{l}0.0019_{0} \\
0.0003_{1}\end{array}$ & $\begin{array}{l}0.0019_{6} \\
0.0002_{0}\end{array}$ & $\begin{array}{l}0.0002_{3} \\
0.0001_{3}\end{array}$ \\
\hline
\end{tabular}

${ }^{*} T$ is the average maximum temperature of a set of observations as given under each of the thermometers in table 4. The subscripts to $T$ define the cells with which the measurements were made.

In connection with the foregoing discussion, it may be of interest to examine more closely the behavior of a cell as illustrated by figure 3 and table 3 . It will be noted that some 35 or 40 minutes elapse before the temperature becomes constant within a few tenths of a millidegree in the normal course of observations. The lag of the thermometer can not account for more than a fraction of this interval, possibly 10 minutes. The remainder of the lag is believed to have the following explanation. Although the solid and liquid phases are brought very close to equilibrium by shaking the cell as the crystals form, a slight departure from equilibrium near the wall of the thermometer well occurs when the thermometer is placed in the well. Loss of heat to the thermometer causes a thin film of solid to form on the wall. Unless the acid is ideally pure this separation of crystals from the now unagitated liquid will lower the purity of the liquid in the region immediately adjacent to and in contact with this thin film of solid. By a process of diffusion, possibly aided by slow convection within the liquid, the composition of this liquid layer gradually approaches that of the bulk of the liquid. As this goes on, the freezing temperature rises until there is no further change in composition. A substantially constant temperature is then maintained for a while. This reasoning is supported by the observations made with thermometer 618 on Sept. 29, 1941 (see table 3), which were obtained under conditions different from normal. The thermometer was warmed at the freezing temperature in an auxiliary cell and then quickly transferred to cell 15. Under these conditions only a small quantity of acid froze around the thermometer well, with a correspondingly smaller change in concentration of impurity in the layer of liquid bounding this solid. It will be noted that in this instance the temperature rose to within a few tenths of a millidegree of the maximum in a much shorter time than usual.

Decline from the maximum temperature, as shown by the curves in figure 3 , comes about from one or both of two causes. One is the increasing concentration of impurities in the liquid as freezing progresses from the outer wall of the cell toward the thermometer well. The other, and possibly the more significant one in this instance, is the lowering of pressure within the cell as freezing progresses. The contraction of the liquid on freezing is greater than the volume of atmospheric gases discharged by the liquid as it freezes. Hence there is a net decrease in pressure and, as will be shown in a later section of this paper, a lowering of the freezing temperature. 
In cells to be prepared in the future it is proposed to use a much lower pressure of gas, so that the effects of a change of pressure during freezing will be minimized. ${ }^{14}$

\section{FREEZING TEMPERATURES OF THE PURE ACID UNDER DEFINED CONDITIONS}

In addition to the group of cells used for the study of the reproducibility of the freezing temperature, one cell was prepared for the measurement of the freezing temperature of the acid under known pressures of oxygen, nitrogen, and a mixture of these gases. The

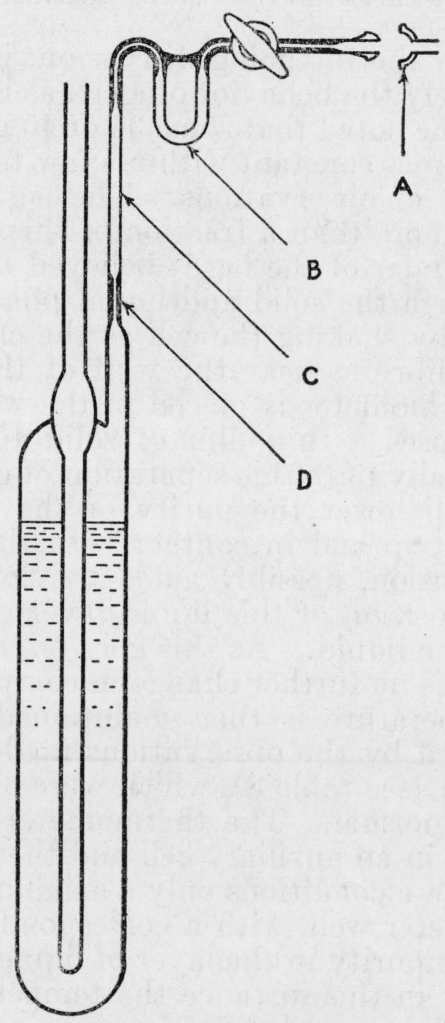

FigURE 4.-Cell used for observations of the freezing temperature under defined conditions.

$A$, Movable ball-joint connection; $B$, trap; $C$, air condenser; $D$, capillary tube (2 mm).

triple point of the acid and the variation of the freezing temperature as a function of pressure were also measured in this cell. The cell, shown in figure 4, differed from the others in that it could be attached to a source of variable pressure of selected gases. The capillary

14 C. S. Cragoe and H. F. Stimson in unpublished observations at the triple point of water, made in a cell similar to that shown in figure 1 , froze a thick layer of ice around the thermometer well and then, by warming the well, melted a thin layer next to it. This provided a surface at equilibrium temperature between ice and liquid water which had been purified by the process of freezing. This surface almost entirely surrounded the thermometer and hence controlled its temperature. This technic may prove advantageous with the benzoic acid cell but has not yet been tried. A similar technic was suggested by W. P. White, J. Am. Chem. Soc. 56, 20 (1934). 
portions of the connecting tube retarded the tendency for benzoic acid to sublime out of the cell when molten, and the larger portions served as condensers for the small amount of vapor that did pass the capillaries. The spherical joint, $A$, made it possible to rock the tube, if desired, while it was connected to the system for supplying gases and measuring the pressure.

When the acid in this cell was to be prepared for an observation, it was melted with a flame rather than in the oven. Care was taken to heat the acid slowly and uniformly so as to avoid overheating it. To get the desired mush of crystals in the liquid, the cell was manipulated in the manner previously described, with the additional detail that capillary $D$ was kept cool, so that crystals of the acid would collect at this point and keep liquid from flowing into the trap or to the stopcock while the cell was being shaken. The cell was kept in a previously warmed Dewar flask during the temperature measurements. Further details of the manipulation required by special conditions of observation will be given in the appropriate paragraphs.

\section{DETERMINATION OF THE TRIPLE POINT}

To prepare for the determination of the triple point the acid was freed of water and atmospheric gases by evacuation of the cell while the acid was allowed to freeze slowly. The melting and freezing were repeated until no further change of pressure occurred when the cell was connected at $A$ (fig. 4) to an evacuated system connected with a mercury manometer. Once the cell was freed of gases in the manner indicated, it did not require attachment to the gas-supply train during the observations of the triple point.

The triple point was measured on three consecutive days and twice more about a month later. The two later measurements were made between series of observations of the freezing temperatures under oxygen and nitrogen. They served as a check on the absence of any significant change in the composition of the acid which might have resulted from the repeated meltings.

The five observations of the triple point are given in table 8 . It will be noted that the range of the five observations, including the two made after several measurements had been made under other conditions, is 0.9 millidegree, and that the changes during the series were not progressive. The small random variations possibly can be ascribed to differences in the fraction of acid frozen, that is, to the fact that observations were not made each time at exactly the same point in the freezing range of the slightly impure acid.

TABLE 8.-Triple point of benzoic acid

\begin{tabular}{|c|c|}
\hline Date & Triple point \\
\hline $\begin{array}{l}\text { Sept. } 23,1941 \\
\text { Sept. 24, } 1941- \\
\text { Sept. } 25,1941- \\
\text { Oct. } 21,1941- \\
\text { Oct. } 24,1941- \\
\end{array}$ & $\begin{array}{l}{ }^{\circ} \mathrm{C} \\
122.31_{8} \\
122.362_{3} \\
122.362_{1} \\
122.361_{4} \\
122.362_{2}\end{array}$ \\
\hline Average....... & 122.3620 \\
\hline
\end{tabular}


The acid used for these measurements was known to contain a small amount of impurity. To make the necessary correction of the triple point, the freezing temperature of the acid in this cell under $1 \mathrm{~atm}$ of dry air was compared with that of acid of known purity also under $1 \mathrm{~atm}$ of dry ajr and observed in another apparatus which will be described in a later section. The freezing temperature of the acid used for the triple-point measurements was 6 millidegrees below that of the pure acid. The amount of impurity, confirmed by a measurement of the freezing range, was found to be 0.0084 mole percent, which corresponds to a lowering of the freezing temperature of 6 millidegrees. This correction has been applied to the temperatures in table 8. By adding an uncertainty of $\cdot 1$ millidegree involved in the triple-point measurements to the uncertainty of 1 millidegree attached to the freezing temperature in dry air of the pure acid, the value of the triple point of pure benzoic acid can be given as $122.362^{\circ}$ $\pm 0.002^{\circ} \mathrm{C}$.

\section{FREEZING TEMPERATURES UNDER DIFFERENT PRESSURES OF OXYGEN AND OF NITROGEN}

To measure the freezing temperatures in equilibrium with selected pressures of oxygen or nitrogen, the acid in the cell was first freed of gases in the manner described in the preceding section. With the acid in the cell at room temperature, gas was admitted at a selected pressure; which, after a few trials, bore a known relation to the pressure which would exist in the cell after it was isolated from the train and the acid had been melted, shaken long enough to insure saturation with gas, brought to the standard conditions for observations of the freezing temperature, placed in a Dewar flask, and then shaken 15 minutes longer to insure equilibrium. When the freezing temperature reached its maximum value, the actual pressure in the cell was checked by setting the manometer to the predicted pressure and then opening the stopcock. The change in the manometer never exceeded a few centimeters. The pressure in the cell was computed from the manometer setting (predicted pressure), the observed pressure after the cell was opened to the manometer, and the known volumes of the gas space in the manometer and in the cell..$^{15}$

The oxygen and nitrogen used for these measurements were, respectively, 99.87 and 99.74 percent pure, as determined from analyses made by Martin Shepherd and Shuford Schuhmann of the Gas Chemistry Section of this Bureau. Both gases contained some argon. Other impurities consisted primarily of oxygen in the nitrogen and of nitrogen in the oxygen. The gases were dried over phosphorus pentoxide but not otherwise purified. Each gas was introduced into the cell in three successive steps, up to a maximum pressure of about $2 \mathrm{~atm}$. Between the two series of measurements, the triple point was redetermined to make sure that no change in the purity of the acid had occurred. The results of the measurements are given in the third column of table 9 and in curves B and D of figure 5. The

15 The ratios of the gas space of the system to the gas space of the cell $v_{s} / v_{c}$ and of the gas space of the manometer to the gas space of the cell $v_{m} / v_{c}$ were found to be 2.17 and 1.17 , respectively. These ratios were constant over the range of pressures measured. The pressure in the cell was computed from the relation

$$
p_{c}=2.17 \mathrm{P},-1.17 p_{m},
$$

in which $p_{e}=$ pressure in the cell, $\mathrm{p}_{m}{ }^{*}=$ pressure $\mathrm{n}$ the manometer and $\mathrm{P}_{\mathrm{s}}=$ pressure in the system, which consists of the cell and the manometer. 


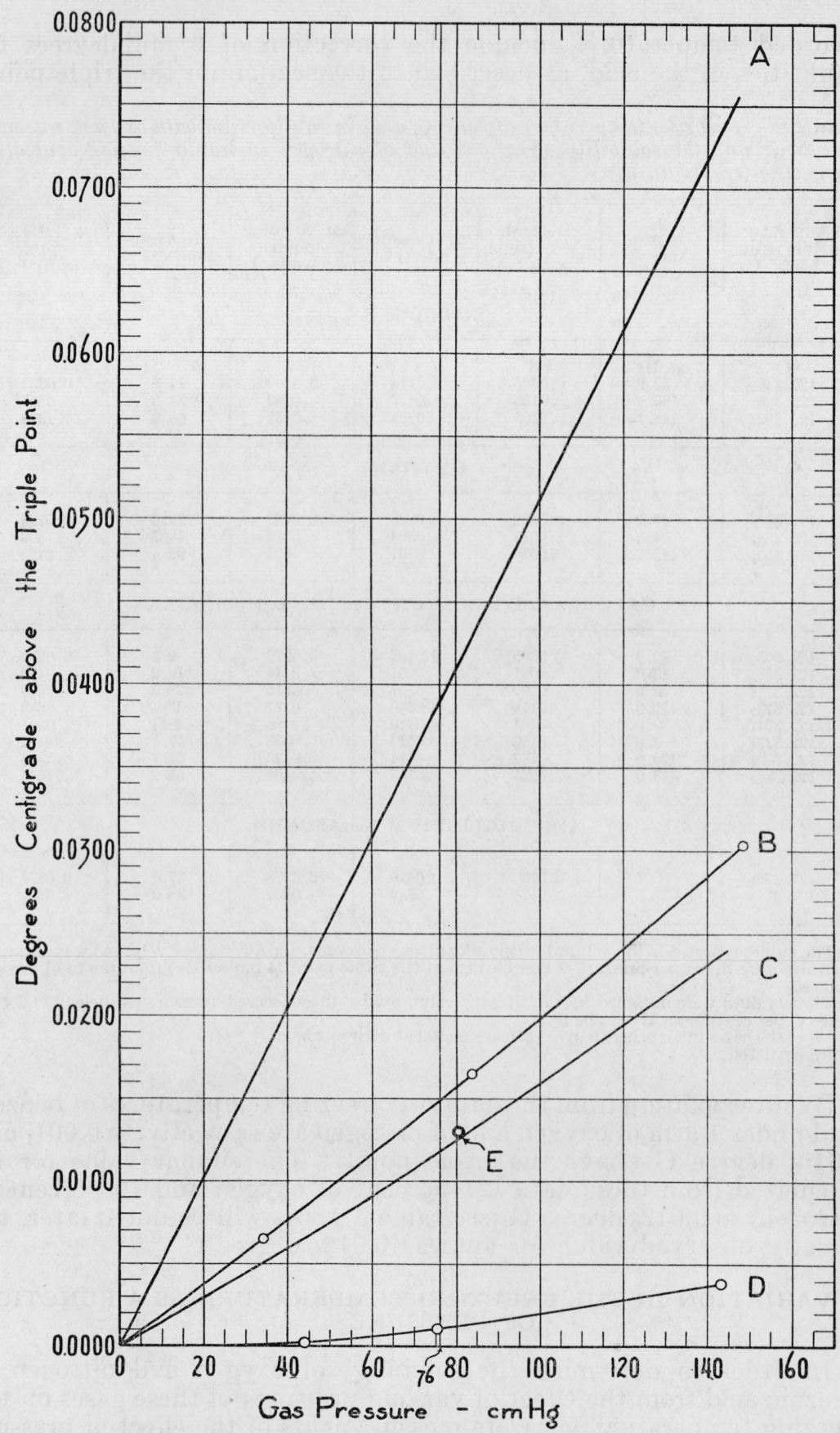

FIGURE 5.-Difference between the triple point and the freezing temperature of benzoic acid in equilibrium with variable pressures of nitrogen, of oxygen, and of air, and the effect of pressure on the freezing temperature.

A, Pressure effect; B, equilibrium with nitrogen; C, equilibrium with air (21 percent of oxygen and 79 percent nitrogen), computed from the observed values for oxygen and for nitrogen; $D$, equilibrium with oxygen; $\mathrm{E}$ observed freezing temperature of benzoic acid $\left(122.375_{2}{ }^{\circ} \mathrm{C}\right)$ in equilibrium with air under a pressure of $80.1 \mathrm{~cm}$ of $\mathrm{Hg}$ (table 9).

$$
634436-45-5
$$


recorded temperatures include the correction of 6 millidegrees for impurities in the acid, as described in the section on the triple point.

TABLE 9. - Freezing temperature of benzoic acid in equilibrium with oxygen, nitrogen, and air and the solubility of oxygen and of nitrogen in liquid benzoic acid at its freezing temperature

\begin{tabular}{|c|c|c|c|c|c|c|}
\hline $\begin{array}{l}\text { Temperature } \\
\text { (triple point } \\
122.3620^{\circ} \mathrm{C} \text { ) }\end{array}$ & $\begin{array}{l}\text { Gas pres- } \\
\text { sure a }\end{array}$ & $\begin{array}{c}\text { Degrees } \\
\text { above triple } \\
\text { point }\end{array}$ & $\begin{array}{c}\Delta t \text { (pressure } \\
\text { effect) b }\end{array}$ & $\begin{array}{c}\Delta t \text { (freezing } \\
\text { point } \\
\text { lowering) }\end{array}$ & $\begin{array}{l}\text { Mole frac- } \\
\text { tion } \times 10^{-5}\end{array}$ & $\begin{array}{l}g(1,000 \mathrm{~g} \\
\text { benzoic } \\
\text { acid })^{-1}\end{array}$ \\
\hline \multicolumn{7}{|c|}{ NITROGEN } \\
\hline $\begin{array}{c}{ }^{\circ} \mathrm{C} \\
122.368_{5} \\
122.378_{5} \\
122.392_{3}\end{array}$ & $\begin{array}{r}c m \text { Hg } \\
33.9 \\
83.4 \\
148.1\end{array}$ & $\begin{array}{l}{ }^{\circ} \mathrm{C} \\
0.0065 \\
.0165 \\
.0303\end{array}$ & $\begin{array}{l}{ }^{\circ} \mathrm{C} \\
0.0174 \\
.0427 \\
.0758\end{array}$ & $\begin{array}{l}{ }^{\circ} \mathrm{C} \\
0.0109 \\
.0262 \\
.0457\end{array}$ & $\begin{array}{l}14.5 \\
34.9 \\
60.9\end{array}$ & $\begin{array}{r}0.0333 \\
.0801 \\
.1398\end{array}$ \\
\hline \multicolumn{7}{|c|}{ OXYGEN } \\
\hline $\begin{array}{l}122.362_{2} \\
122.363_{1} \\
122.365_{8}\end{array}$ & $\begin{array}{r}43.6 \\
75.0 \\
142.7\end{array}$ & $\begin{array}{r}0.0002 \\
.0011 \\
.0038\end{array}$ & $\begin{array}{r}0.0223 \\
.0384 \\
.0731\end{array}$ & $\begin{array}{r}0.0221 \\
.0373 \\
.0693\end{array}$ & $\begin{array}{l}29.5 \\
49.7 \\
92.4\end{array}$ & $\begin{array}{r}0.0773 \\
.1303 \\
.2423\end{array}$ \\
\hline \multicolumn{7}{|c|}{ AIR CALCULATED FROM OXYGEN AND NITROGEN • } \\
\hline $\begin{array}{l}122.365_{0} \\
122.368_{1} \\
122.371_{2} \\
122.373_{8} \\
122.374_{4} \\
122.377_{6} \\
122.380_{9} \\
122.384_{3}\end{array}$ & $\begin{array}{r}20.0 \\
40.0 \\
60.0 \\
76.0 \\
80.0 \\
100.0 \\
120.0 \\
140.0\end{array}$ & $\begin{array}{r}0.0030 \\
.0061 \\
.0092 \\
.0118 \\
.0124 \\
.0156 \\
.0189 \\
.0223\end{array}$ & $\begin{array}{r}0.0102 \\
.0205 \\
.0307 \\
.0389 \\
.0410 \\
.0512 \\
.0614 \\
.0717\end{array}$ & $\begin{array}{r}0.0072 \\
.0144 \\
.0215 \\
.0271 \\
.0286 \\
.0356 \\
.0425 \\
.0494\end{array}$ & $\begin{array}{r}9.6 \\
19.2 \\
28.7 \\
36.1 \\
38.1 \\
47.5 \\
56.7 \\
65.9\end{array}$ & $\begin{array}{r}0.0226 \\
.0452 \\
.0678 \\
.0853 \\
.0901 \\
.1122 \\
.1340 \\
.1558\end{array}$ \\
\hline \multicolumn{7}{|c|}{ AIR-EQUILIBRIUM MEASURED } \\
\hline $\begin{array}{l}122.375_{2} \\
122.374_{5}\end{array}$ & $\begin{array}{r}80.1 \\
\text { d } 76.0\end{array}$ & $\begin{array}{r}0.0132 \\
.0125\end{array}$ & $\begin{array}{r}0.0410 \\
.0389\end{array}$ & $\begin{array}{r}0.0278 \\
.0264\end{array}$ & $\begin{array}{l}37.1 \\
35.2\end{array}$ & $\begin{array}{r}0.0876 \\
.0832\end{array}$ \\
\hline
\end{tabular}

a The values reported in this column are those for nitrogen, oxygen, and air, respectively, and were obtained by subtracting the vapor pressure of benzoic acid at the triple point ( $4 \mathrm{~mm}$ of $\mathrm{Hg}$ ) from the total pressure observed.

b These values were obtained by multiplying column 2 by the observed pressure coefficient, $51.2 \times 10^{-5}$ degree $\mathrm{C}$ per centimeter Hg (table 10).

All of the gases in air other than oxygen are included with nitrogen.

d Interpolated.

By interpolation from the data, the freezing temperatures of benzoic acid under $1 \mathrm{~atm}$ of oxygen and of nitrogen are respectively $0.001_{1}$ and $0.015_{0}$ degree $\mathbf{C}$ above the triple point. The similar value for air calculated from these data (21 percent of oxygen and 79 percent of nitrogen) is $0.012_{0}$ degree $\mathrm{C}$ (see table 9). As will be noted later, the directly observed value for air was $0.012_{5}$ degree C.

\section{VARIATION IN THE FREEZING TEMPERATURE AS A FUNCTION OF PRESSURE}

In order to determine the solubility of oxygen and nitrogen in benzoic acid from the effect of varying pressures of these gases on the freezing temperature, separate measurements of the effect of pressure were necessary. These were made as follows: When certain of the observations of freezing temperature in equilibrium with measured pressures of a gas were completed, the pressure of the gas in the cell was suddenly changed to some higher value. This produced an instan- 
taneous change in pressure but did not for some time alter the concentration of gas in the portion of the liquid around the thermometer well, especially in the region near the thermometer coil. This lag in the change of concentration of the gas was caused by the long path through which gases dissolving at the upper surface of the liquid would have to diffuse to reach the level of the thermometer coil. Care was taken not to agitate the cell during these observations. The change in temperature caused by the change in pressure usually was complete within 3 minutes and always within 5 . The change was shown to be reversible by observations made when the sign of the pressure change was reversed. The observed effects of these changes in pressure are given in table 10 . In the first column of table 10 is given the pressure in centimeters before and after the pressure was suddenly changed. The pressure interval is given in column 2 and the corresponding change in observed temperature in column 3 . The changes in freezing temperatures for a change in pressure of $1 \mathrm{~cm}$ and of $1 \mathrm{~atm}$, as calculated from the observed values, are given in columns 4 and 5, all of table 10. The pressure coefficient is $0.038_{9}^{\circ} \mathrm{C}$ per atmosphere, as shown in curve $A$ of figure 5 .

TABLE 10.-Effect of pressure on the freezing temperature of benzoic acid

\begin{tabular}{|c|c|c|c|c|}
\hline Pressure & $\begin{array}{l}\text { Change of } \\
\text { pressure } \\
\Delta P\end{array}$ & $\Delta T$ & $\Delta T / \Delta P \times 10^{-5}$ & $\begin{array}{l}\text { Change of } \\
\text { temperature } \\
\text { per atmos- } \\
\text { phere }\end{array}$ \\
\hline \multirow{5}{*}{$\begin{array}{c}c m \text { Hg. } \\
116.7 \\
147.0 \\
60.2 \\
\text { Average... }\end{array}$} & $\mathrm{cm} \mathrm{Hg}$ & ${ }^{\circ} \mathrm{C}$ & ${ }^{\circ} \mathrm{C} / \mathrm{cm} \mathrm{Hg}$ & ${ }^{\circ} \mathrm{C} / \mathrm{atm}$ \\
\hline & 56.8 & $0.028_{0}$ & 50.4 & $0.038_{3}$ \\
\hline & 30.3 & $.015_{6}$ & 51.5 & $.039_{1}$ \\
\hline & -86.8 & $-.044_{8}$ & +51.6 & $+.039_{2}$ \\
\hline & & & 51.2 & $.038_{\theta}$ \\
\hline
\end{tabular}

\section{SOLUBILITIES OF OXYGEN AND OF NITROGEN IN BENZOIC ACID}

In column 4 of table 9 are given the interpolated values of the effect of pressure corresponding to the pressures at which the system was observed in equilibrium with oxygen and nitrogen. By subtracting the "elevation of the triple point" (column 3 ) caused by a given pressure of gas at equilibrium from the corresponding elevation caused by pressure as such (column 4), the depression of the freezing temperature caused by the dissolved gas is obtained. These values are given in column 5. The corresponding mole fractions of dissolved gas given in column 6 were calculated on the basis of the equation ${ }^{16}$

$$
x=\frac{\Delta t L_{f_{0}}}{R T_{f_{0}}^{2}}
$$

in which $x$ is the mole fraction of the solute; $T_{f_{0}}$ is the freezing point of the pure substance on the Kelvin scale; $\Delta t=T_{f_{0}}-T_{f}$, where $T_{f}$ is the initial freezing temperature (the temperature at which an

16 Frank W. Schwab and Edward Wichers, Precise Measurement of the Freezing Range as a Means of Determining the Purity of a Substance, Temperature-Its Measurement and Control in Science and Industry, pp. 256-264 (Reinhold Publishing Corporation, New York, N. Y., 1941). 
infinitely small quantity of solid is in equilibrium with the solution); $L_{f_{0}}$ is the molal heat of fusion of the pure substance; and $R$ is the gas constant. The solubility under a pressure of $1 \mathrm{~atm}$, also shown in figure 6 , is $0.132_{1}$ and $0.073_{2} \mathrm{~g}$ of oxygen and of nitgrogen, respectively, per thousand grams of acid. From figure 5 it will be seen that the solubility of each gas appears to increase slightly less than in direct proportion to its increase in pressure. It is believed that the measurements were made with sufficient accuracy to justify this conclusion. In view of the slight bearing of these observations on the principal

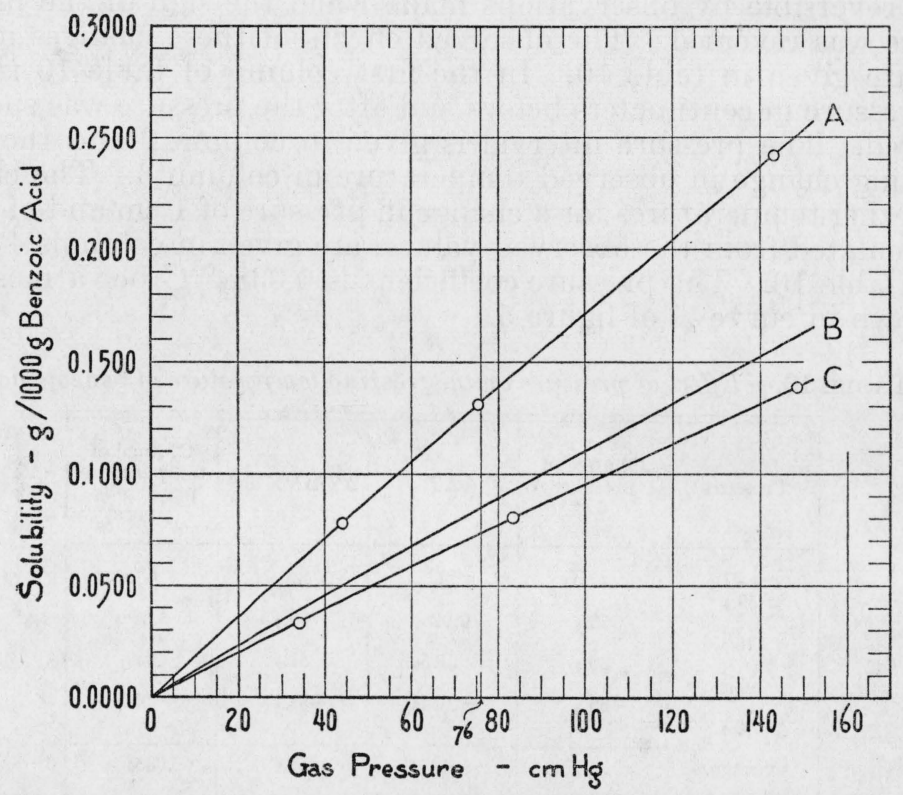

FIGURE 6.-Solubility of oxygen, nitrogen, and air in benzoic acid at the freezing temperature.

A, Solubility of oxygen, determined; B, solubility of air (21 percent oxygen and 79 percent nitrogen), computed from the determined solubility of oxygen and of nitrogen (table 9); C, solubility of nitrogen, determined.

object of this investigation, it did not appear justifiable to spend the time which would have been required to check the results by direct measurements of solubility.

\section{FREEZING TEMPERATURE UNDER ONE ATMOSPHERE OF DRY AIR}

The apparatus shown in figure 4 was used to determine the freezing temperature of the acid in equilibrium with air. The side arm of the cell was cut below $D$ and a fritted-glass bubbler, which extended to the bottom of the cell, was inserted. It was attached with a ring seal arranged so that air could be forced into the liquid and out of the cell through a stopcock open to the atmosphere. Although liquid and solid acid were in the cell in about the same proportions as those existing during observations of the freezing temperature, dry air was passed through the cell long enough to insure a close approach to equilibrium 
with dissolved oxygen and nitrogen under a combined pressure of 1 $\mathrm{atm}$. The stopcock was then closed and the freezing temperature observed in the usual way. The freezing temperature was $122.375_{2}{ }^{\circ} \mathrm{C}$, and the simultaneously measured pressure in the cell was $80.5 \mathrm{~cm}$ of mercury (table 9 and fig. 5). During these observations the temperature of the upper end of the cell was probably slightly higher than it was while the air was being swept through the cell, hence the pressure rose slightly above $1 \mathrm{~atm}$. The freezing temperature corrected to $1 \mathrm{~atm}$ was $122.374_{5}^{\circ} \mathrm{C}$.

To determine the freezing temperature of benzoic acid in equilibrium with air in another manner, a different form of apparatus was used. This apparatus, which has been described in an earlier publication ${ }^{17}$ is shown in figure 7. It was designed for measuring freezing ranges. The thermometer was centered in a cylindrical glass freezing tube 2 $\mathrm{cm}$ in diameter and about $25 \mathrm{~cm}$ long, provided with a glass jacket that could be evacuated if desired. The thermometer was supported by a ground-in glass head provided with an outlet tube for the passage of air from the freezing-range cell. The tip of the thermometer was about $2 \mathrm{~cm}$ from the bottom of the cell, and the depth of the liquid in the cell was $20 \mathrm{~cm}$. Just above the bottom of the cell was a glass bubbler of the type described by Branham and Sperling ${ }^{18}$ with orifices about 0.003 in. in diameter. The bubbler was provided with an inlet tube passing through the double-walled vessel and connected to a source of purified air dried over phosphorus pentoxide or conditioned to contain a desired partial pressure of water.

The freezing temperature of a portion of acid of high purity was measured as follows: While the acid was being melted in the apparatus it was stirred with air saturated with water at $0^{\circ} \mathrm{C}$. It was then brought to a temperature about 10 degrees above the freezing temperature and thoroughly treated with air saturated with water at $-25^{\circ} \mathrm{C}$. Air saturated at this temperature contains a partial pressure of water vapor of about $0.5 \mathrm{~mm}$ of mercury, which is 10 times as much as is needed to cause virtually complete reversion of benzoic anhydride to benzoic acid. ${ }^{19}$ The cell was then transferred to an oil bath that was kept 8 degrees below the freezing point of the acid. The acid undercooled about 0.5 degree C. When crystals suddenly formed, the temperature rose to within a few millidegrees of the equilibrium temperature in less than 2 minutes. About 5 percent of the acid was allowed to freeze, and then the temperature of the oil bath was quickly raised to the freezing temperature of the acid and kept constant within 0.01 degree C. This prevented further formation of solid and allowed the thermometer to indicate the temperature of equilibrium. The freezing temperature under these conditions was $122.369_{9}^{\circ} \mathrm{C}$. Next, the acid was stirred with air dried over phosphorus pentoxide until the temperature rose to a constant value at $122.374_{0}^{\circ} \mathrm{C}$, representing equilibrium in contact with dry air. This required 40 minutes, and observations were continued for 30 minutes longer. Thereafter the air used for stirring contained a partial pressure of water vapor of $4.6 \mathrm{~mm}$ of $\mathrm{Hg}$, corre-

\footnotetext{
17 Frank W. Schwab and Edward Wichers, Precise Measurement of the Freezing Range as a Means of Determining the Purity of a Substance, Temperature-Its Measurement and Control in Science and Industry, p. 256-264 (Reinhold Publishing Corporation, New York, N. Y., 1941).

18 J. R. Branham and E. O. Sperling, Bubbler tip of Pyrex glass for difficult absorptions, J. Research NBS 22, 701 (1939) RP1214.

19 Frank W. Schwab and Edward Wichers, Preparation of ben zoic acid of high purity, J. Research NBS 25 , 747 (1940) RP1351.
} 


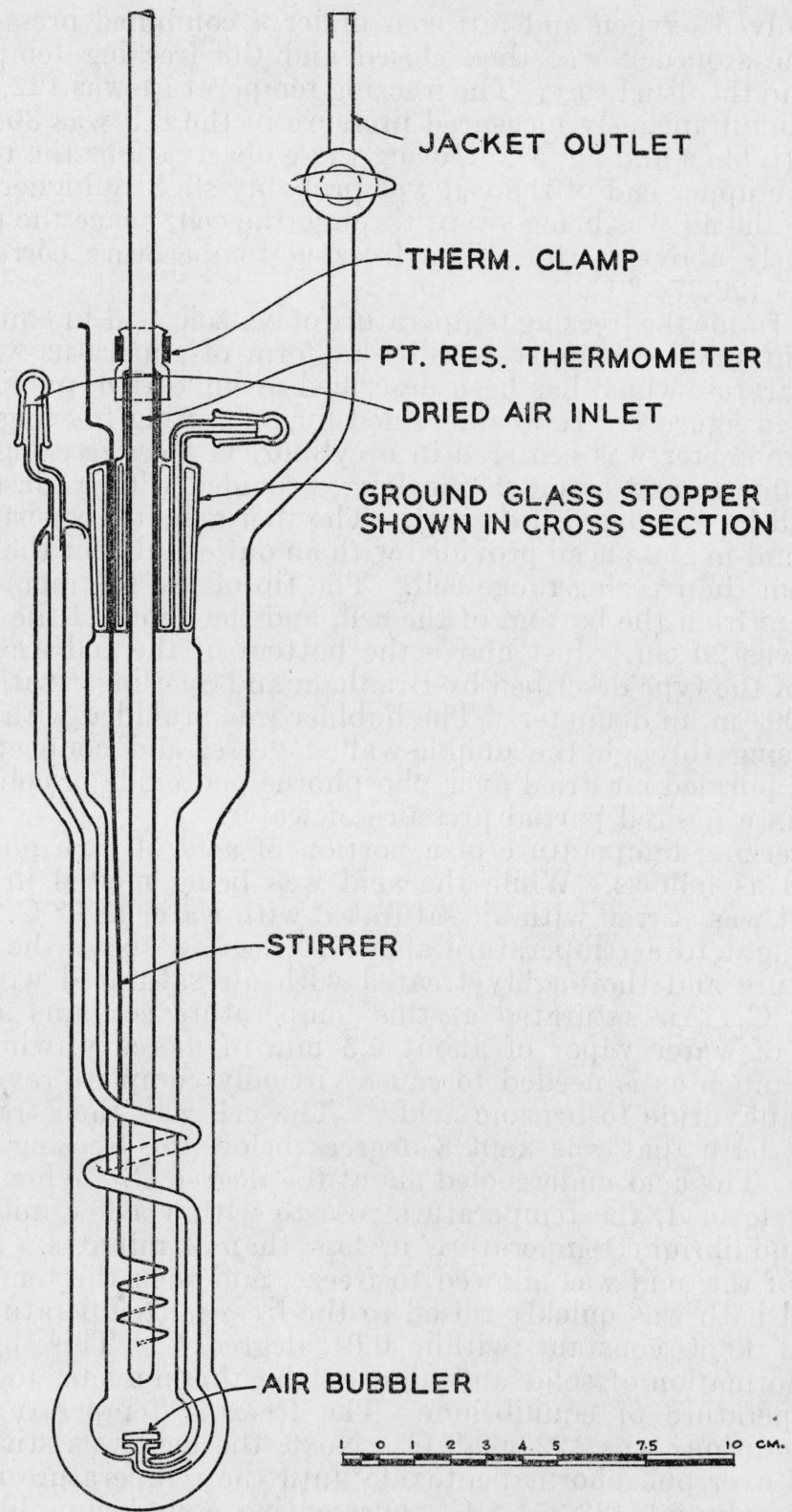

Frgure 7.-Apparatus for measuring the freezing temperature in equilibrium with dry air and for determining the purity of the acid.

sponding to saturation with water vapor at $0^{\circ} \mathrm{C}$, whereupon the freezing temperature fell to $122.343_{8}{ }^{\circ} \mathrm{C}$. The two temperatures observed, when the mixture of solid and liquid acid was in equilibrium with air containing partial pressures of water vapor of 4.6 and $0.5 \mathrm{~mm}$ of $\mathrm{Hg}$, offered a means of extrapolating to the freezing temperature in 
equilibrium with dry air. The extrapolated temperature was $122.373^{\circ}$ $\mathrm{C}$, which agrees sufficiently well with the observed temperature under dry air to indicate that the acid, when thus observed, did not contain a significant amount of benzoic anhydride.

After these observations were completed the purity of the acid was determined by measuring its freezing range in the same apparatus by a procedure previously described. ${ }^{20}$ Its purity was found to be $99.998_{4}$ mole percent. The presence of $0.001_{6}$ mole percent of impurity caused an initial depression of the freezing temperature of $0.001_{2}$ degree $\mathrm{C}$, or of $0.001_{3}$ degree $\mathrm{C}$ at the point in the freezing range when 5 percent of the substance is frozen. This correction was therefore applied to the observed freezing temperature of the acid $\left(122.374_{0}^{\circ} \mathrm{C}\right)$, giving a value for pure benzoic acid of $122.375_{3}{ }^{\circ} \mathrm{C}$, with an estimated uncertainty of \pm 0.001 degree $\mathrm{C}$.

\section{CHANGE IN THE SPECIFIC VOLUME OF BENZOIC ACID THAT ACCOMPANIES FREEZING}

The change in volume when benzoic acid freezes is of interest in connection with its behavior in the sealed cells. No data were found on the density of solid benzoic acid at or near its freezing point, and no apparently reliable values for the density of the liquid at that temperature. Observations by Timmermans and Burriel ${ }^{21}$ on the liquid at $130^{\circ}, 155^{\circ}$, and $180^{\circ} \mathrm{C}$ permit extrapolation to the freezing temperature. The respective values for the density are 1.07494, 1.05218 , and $1.02942 \mathrm{~g} / \mathrm{ml}$. Extrapolation to the freezing temperature gives $1.0819 \mathrm{~g} / \mathrm{ml}$. In this calculation the freezing temperature given by Timmermans $\left(122.45^{\circ} \mathrm{C}\right)$ was used so as to minimize the effect of possible systematic errors in the other temperatures.

There are no data for the density of the solid acid at temperatures higher than $23.3^{\circ} \mathrm{C}$. The only available coefficient of expansion ${ }^{22}$ is for the interval between $15^{\circ}$ and $30^{\circ} \mathrm{C}$. This value is probably in error because its calculation involved Lumsden's ${ }^{23}$ determination of the density at $15^{\circ} \mathrm{C}\left(1.266 \mathrm{~g} / \mathrm{cm}^{3}\right)$, a value which appears to be less accurate than the one published by Hendricks and Jefferson ${ }^{24}$ $\left(1.3211 \pm 0.0001 \mathrm{~g} / \mathrm{cm}^{3}\right.$ at $\left.23.3^{\circ} \mathrm{C}\right)$. It was accordingly impossible to make any reasonably good extrapolation to the density of the solid acid at its melting point. The change of volume on freezing was, therefore, calculated from the recorded value for the heat of fusion per gram $\Delta H_{f}, 1,400 \mathrm{cc}$-atm, the observed pressure coefficient of the freezing temperature $\Delta T / \Delta P, 0.038_{9}^{\circ} \mathrm{C} / \mathrm{atm}$, and the freezing temperature of the acid $T, 395.6^{\circ} \mathrm{K}$, by means of the ClausiusClapeyron equation

$$
\frac{\Delta P}{\Delta T}=\frac{\Delta H_{f}}{T\left(v_{2}-v_{1}\right)},
$$

20 Frank W. Schwab and Edward Wichers, Precise Measurement of the Freezing Range as a Means of Determining the Purity of a Substance, Temperature-Its Measurement and Control in Science and Industry, p. 256-264 (Reinhold Publishing Corporation, New York, N. Y., 1941).

21 Jean Timmermans and F. Burriel, Recherches sur les propriétés de quélques combinaisons organiques, solides a la temperature ordinaire, Chimie \& Industrie, Special No., p. 196-7, March (1931).

22 E. R. Smith, The determination of the coefficient of cubical expansion of solid benzoic acid by means of a gas-filled dilatometer, BS J. Research 7,903 (1931) RP382.

${ }_{23}$ John Scott Lumsden, The physical properties of heptoic, hexahydrobenzoic, and benzoic acids and their derivatives, J. Chem. Soc. 87,90 (1905).

24 S. B. Hendricks and M. E. Jefferson, Anisotropy of molecular crystals. I. Experimental, J. Optical Soc. of America 23, 299 (1933). 
in which $v_{1}$ is the specific volume of the liquid form, and $v_{2}$ that of the solid. The shrinkage which occurs on freezing, thus calculated, is $0.138 \mathrm{~cm}^{3} / \mathrm{g}$. The corresponding calculated value for the density of the solid at the freezing temperature is $1.27 \mathrm{~g} / \mathrm{cm}^{3}$.

The foregoing calculated value for the density at $122.37^{\circ} \mathrm{C}$ and the value of Hendricks and Jefferson for the density at $23.3^{\circ} \mathrm{C}$ give as the coefficient of cubical expansion, for the 99-degree interval, 0.00040 per degree.

Acknowledgment is made for the advice and many helpful suggestions received from members of the Heat Division, in particular E. F. Mueller, C. S. Cragoe, H. F. Stimson, and C. H. Meyers. Thanks are also expressed to C. L. Gordon for preparing the drawings.

\title{
VII. APPENDIX
}

\section{Calculation of Temperatures in Platinum Resistance Thermometry}

\author{
By Frank W. Schwab and E. R. Smith
}

The Callendar equation has been used for more than a half century for the computation of temperatures. During all these years there was little need for a departure from its use, but recently when it was appreciated that precision platinum resistance thermometers might be calibrated at temperatures other than the ice, steam, sulfur, and oxygen points, it became clear that a direct application of the fundamental equations offers advantages.

The fundamental equations can be modified in a regular manner to improve their application to the computation of temperatures. For the range from $0^{\circ}$ to $660^{\circ} \mathrm{C}$, one modification of the fundamental equation is described in this section. In the range from $-190^{\circ}$ to $0^{\circ}$ $\mathrm{C}$, two forms are given, of which the second is the more suitable for solution by the use of a slide rule.

\section{RELATION BETWEEN THE POWER SERIES, CALLENDAR, AND CALLENDAR-VAN DUSEN EQUATIONS}

The international scale of temperature between $0^{\circ}$ and $660^{\circ} \mathrm{C}$ is defined by the resistance $R_{t}$ of a platinum resistance thermometer by means of the formula

$$
R_{t}=R_{0}\left(1+A t+B t^{2}\right)
$$

for which the constants, $R_{0}, A$, and $B$ are determined from measurements of resistances at the melting point of ice, the boiling point of water, and the boiling point of sulfur. From $-190^{\circ}$ to $0^{\circ} \mathrm{C}$, the temperature scale is obtained from the resistance by the formula

$$
R_{t}=R_{0}\left[1+A t+B t^{2}+C(t-100) t^{3}\right]
$$

in which the constants $R_{0}, A$, and $B$ are the same as in formula 1 and the constant $C$ is found from a measurement of resistance at the boiling point of oxygen. ${ }^{25}$

${ }^{25}$ Compt. rend., Eighth General Conference of Weights and Measures, (Paris, 1933). 
National Bureau of Standards certificates for platinum resistance thermometers report only values for the constants $R_{0}, c$, and $\delta$ for the Callendar formula

$$
t=100 \frac{R_{t}-R_{0}}{R_{100}-R_{0}}+\delta\left(\frac{t}{100}-1\right) \frac{t}{100},
$$

in which the value to be used for $\left(R_{100}-R_{0}\right)$ is given by

$$
\left(R_{100}-R_{0}\right)=100 c R_{0} \text {. }
$$

The value of the constant $\beta$ may also be given for use in the CallendarVan Dusen equation

$$
t=100 \frac{R_{t}-R_{0}}{R_{100}-R_{0}}+\delta\left(\frac{t}{100}-1\right) \frac{t}{100}+\beta\left(\frac{t}{100}-1\right)\left(\frac{t}{100}\right)^{3}
$$

at temperatures below $0^{\circ} \mathrm{C}$.

Equations 1 and 2 are the fundamental international equations. The constants for them can be computed from those of the equivalent forms of the Callendar and Callendar-Van Dusen equations. $R_{0}$, of course, remains the same for all. The relationships between the constants are readily obtained by writing the complete fundamental equation, 2 , in the form

$$
\frac{R_{t}}{R_{0}}=1+A t+B t^{2}+C(t-100) t^{3}
$$

and the Callendar-Van Dusen equation in the equivalent form

$$
\frac{R_{t}}{R_{0}}=1+c\left(1+\frac{\delta}{100}\right) t-\left(\frac{c \delta}{10^{4}}\right) t^{2}-\left(\frac{c \beta}{10^{8}}\right)(t-100) t^{3}
$$

Since these equations are equivalent, comparison of coefficients gives

$$
A=c\left(1+\frac{\delta}{100}\right), B=-\frac{c \delta}{10^{4}}, C=\frac{-c \beta}{10^{8}} .
$$

The constants of the Callendar and Callendar-Van Dusen equations in terms of those of the fundamental equations are

$$
c=A+100 B, \delta=-\frac{10^{4} B}{c}, \beta=-\frac{10^{8} C}{c} .
$$

\section{INTERPOLATION OF TEMPERATURES BY USE OF THE POWER SERIES EQUATIONS}

The power series equations can be expanded to make them more useful for the computation of temperatures by successive approximations. A simple graph together with a short table obviate more than one computation for a precision of the temperature within $0.001^{\circ} \mathrm{C}^{26}$

${ }^{26}$ All of the precisions of the computation of temperature referred to in this appendix are dependent only on the method of computation. Other errors, such as errors of calibration, have been discussed by Brickwedde and Hoge, and others. Harold J. Hoge and Ferdinand G. Brickwedde, Intercomparison of platinum resistance thermometers between $-190^{\circ}$ and $445^{\circ} \mathrm{C}$, J. Research NBS 28, 217 (1942) RP1454. 
(a) IN THE RANGE FROM $0^{\circ}$ TO $660^{\circ} \mathrm{C}$

With a calculating machine, the fundamental equations can be used as conveniently as the Callendar and Callendar-Van Dusen equations to compute temperatures from resistances. For example, equation 1 can be written in the form

$$
\left(\frac{R_{t}}{R_{0}}-1\right)=t(A+B t) \text { and } t=\frac{\left(R_{t} / R_{0}\right)-1}{A+B t}
$$

If $t_{1}$ is a first estimation of the temperature,

etc.

$$
t_{2}=\frac{\left(R_{t} / R_{0}\right)-1}{A+B t_{1}}
$$

When the measured temperature is between $0^{\circ}$ and $100^{\circ} \mathrm{C}$, and the first estimated temperature, $t_{1}$, is correct to within $1^{\circ} \mathrm{C}$, one solution of equation 9 yields the correct temperature to the nearest $0.01^{\circ} \mathrm{C}$. In the range between $100^{\circ}$ and $660^{\circ} \mathrm{C}$, two successive solutions are sufficient for this accuracy.

Better convergence can be obtained by the formula

$$
t_{2}=\frac{\left[\left(R_{t} / R_{0}\right)-1\right]+B t_{1}^{2}}{A+2 B t_{1}}
$$

which can be derived from equation 1 by the Newton-Raphson method of series approximation ${ }^{27}$ or, less elegantly, by adding $R_{0} B t^{2}$ to each side of equation 1 . With equation 10 , if the first estimate of the temperature, $t_{1}$, is correct to within $2^{\circ} \mathrm{C}$, the first solution of the equation for $t_{2}$ yields the temperature correctly to within $0.001^{\circ} \mathrm{C}$ over the complete range of validity of equation 1 . The first estimation for $t_{1}$ can be made to within $2^{\circ} \mathrm{C}$ from a rough plot of $R_{t}$ with respect to $t$. To make this plot, values of $R_{t}$ for $t=0^{\circ}, 100^{\circ}, 200^{\circ} \ldots, 600^{\circ} \mathrm{C}$, can be quickly tabulated by little more than inspection of the fundamental formula $R_{t}=R_{0}\left(1+A t+B t^{2}\right)$. The results are plotted on a scale such that $1 \mathrm{~mm}=1$ degree and $0.1 \mathrm{ohm}$ for a 25 -ohm thermometer. With sufficient accuracy the plot is taken as linear over each 100-degree interval. Then only one computation with equation 10 is needed to find the correct temperature to within the precision of calibration of a platinum resistance thermometer.

(b) IN THE RANGE FROM $-190^{\circ}$ TO $0^{\circ} \mathrm{C}$.

Equation 2 can be solved approximately to obtain

$$
t_{2}=\frac{\left(R_{t} / R_{0}\right)-1}{A+B t_{1}+C\left(t_{1}-100\right) t_{1}^{2}}
$$

for temperatures below $0^{\circ} \mathrm{C}$. For measurements with a $25-\mathrm{ohm}$ thermometer and with an initial estimate of the temperature made correctly to within $1^{\circ} \mathrm{C}$, the first temperature computed by equation 11 differs from the correct temperature by $0.003^{\circ} \mathrm{C}$ at $-25^{\circ} \mathrm{C}$ and by

${ }^{27}$ See also, Scarborough, Numerical Mathematical Analysis, p. 178, Johns Hopkins Press (1930). 
$0.057^{\circ} \mathrm{C}$ at $-190^{\circ} \mathrm{C}$. The second computation, using the first as the estimated value, is correct to better than $0.001^{\circ} \mathrm{C}$ between $0^{\circ}$ and $-140^{\circ} \mathrm{C}$ and is in error by only $0.004^{\circ} \mathrm{C}$ at $-190^{\circ} \mathrm{C}$.

When equation 2 is treated by the Newton-Raphson method, there is obtained

$$
t_{2}=\frac{\left[\left(R_{t} / R_{0}\right)-1\right]+B t_{1}^{2}+C\left(3 t_{1}-200\right) t_{1}^{3}}{A+2 B t_{1}+C\left(4 t_{1}-300\right) t_{1}^{2}} .
$$

This equation appears unwieldly but is quite simple to use with the aid of a modern calculating machine and will yield over the complete range of $0^{\circ}$ to $-190^{\circ} \mathrm{C}$ temperatures correct to the nearest $0.001^{\circ} \mathrm{C}$ on the first computation when the initial estimate, $t_{1}$, is correct to within 2 degrees. If $0.005^{\circ}$ to $0.01^{\circ} \mathrm{C}$ is sufficient precision, the initial estimate need be correct to within $5^{\circ} \mathrm{C}$.

An equation of the same form as equation 12 but a little simpler in the final terms of the numerator and denominator can be found by adding $B t^{2}+\mathrm{nC}(t-100) t^{3}$ to each side of equation 2, written in the form $\left(R_{t} / R_{0}\right)-1=A t+B t^{2}+C(\mathrm{t}-100) t^{3}$. Then, on solving approximately for $t$, there is obtained

$$
t_{2}=\frac{\left[\left(R_{t} / R_{0}\right)-1\right]+B t_{1}^{2}+n C\left(t_{1}-100\right) t_{1}^{3}}{A+2 B t_{1}+(n+1) C\left(t_{1}-100\right) t_{1}^{2}} .
$$

By trial, it has been found that equation 13 gives as satisfactory convergence as equation 12 when $n$ is assigned the value of 2.64 , so that equation 13 becomes

$$
t_{2}=\frac{\left[\left(R_{t} / R_{0}\right)-1\right]+B t_{1}^{2}+E\left[\left(t_{1} / 100\right)-1\right] t_{1}^{3}}{A+2 B t_{1}+F\left[\left(t_{1} / 100\right)-1\right] t_{1}^{2}},
$$

in which $E=264 \mathrm{C}$ and $F=364 \mathrm{C}$.

The following procedure is recommended for the calculation of temperatures by means of either equation 12 or 14 .

\section{PROCEDURE FOR THE CALCULATION OF TEMPERATURES BY USE OF THE POWER SERIES EQUATIONS]}

Obtain the initial estimate of the temperature, correct to within $2^{\circ} \mathrm{C}$ from a very simple graph of $R$ plotted with respect to $t$. This graph is made to a scale of $1 \mathrm{~mm}=1^{\circ} \mathrm{C}$ and $0.1 \mathrm{ohm}$. The point for $t=-190^{\circ}$ is connected by a straight line to the point for $t=-100^{\circ}$. The latter is connected by another straight line to the point for $t=0^{\circ}$. The temperature corresponding to any resistance in the range covered can be read from the plot to within $2^{\circ} \mathrm{C}$ for use as $t_{1}$ with either equation 12 or 14 . If equation 12 is to be used, values of the temperature functions useful for a quick computation are taken from table 11. If equation 14 is preferred, table 12 is used. In either case the following method is rapid. 
364 Journal of Research of the National Bureau of Standards

TABLE 11.-Data for computing temperatures in platinum resistance thermometry by the equations ${ }^{1}$

. and

$$
t_{2}=\frac{\left[\left(R_{t} / R_{0}\right)-1\right]+B t_{1}^{2}}{A+2 B t_{1}}
$$

$$
t_{2}=\frac{\left[\left(R_{t} / R_{0}\right)-1\right]+B t_{1}^{2}+C\left(3 t_{1}-200\right) t_{1}^{3}}{A+2 B t_{1}+C\left(4 t_{1}-300\right) t_{1}^{2}}
$$

\begin{tabular}{|c|c|c|c|c|}
\hline - & $-\frac{t}{10^{6}}$ & $-(4 t-300) \frac{t^{2}}{10^{12}}$ & $\frac{t^{2}}{10^{7}}$ & $(3 t-200) \frac{t^{3}}{10^{12}}$ \\
\hline $\begin{array}{l}0 \\
1 \\
2 \\
3 \\
4\end{array}$ & $\begin{array}{r}0.000000 \\
1 \\
2 \\
3 \\
4\end{array}$ & $\begin{array}{r}0.000000000 \\
0 \\
1 \\
3 \\
5\end{array}$ & $\begin{array}{r}0.0000000 \\
1 \\
4 \\
9 \\
16\end{array}$ & $\begin{array}{r}0.000000000 \\
0 \\
2 \\
6 \\
14\end{array}$ \\
\hline $\begin{array}{l}5 \\
6 \\
7 \\
8 \\
9\end{array}$ & $\begin{array}{l}5 \\
6 \\
7 \\
8 \\
9\end{array}$ & $\begin{array}{r}8 \\
12 \\
16 \\
21 \\
27\end{array}$ & $\begin{array}{l}25 \\
36 \\
49 \\
64 \\
81\end{array}$ & $\begin{array}{r}27 \\
47 \\
76 \\
115 \\
165\end{array}$ \\
\hline $\begin{array}{l}10 \\
11 \\
12 \\
13 \\
14\end{array}$ & $\begin{array}{l}10 \\
11 \\
12 \\
13 \\
14\end{array}$ & $\begin{array}{l}34 \\
42 \\
50 \\
59 \\
70\end{array}$ & $\begin{array}{l}100 \\
121 \\
144 \\
169 \\
196\end{array}$ & $\begin{array}{l}230 \\
310 \\
408 \\
525 \\
664\end{array}$ \\
\hline $\begin{array}{l}15 \\
16 \\
17 \\
18 \\
19\end{array}$ & $\begin{array}{l}15 \\
16 \\
17 \\
18 \\
19\end{array}$ & $\begin{array}{r}81 \\
93 \\
106 \\
121 \\
136\end{array}$ & $\begin{array}{l}225 \\
256 \\
289 \\
324 \\
361\end{array}$ & $\begin{array}{r}827 \\
1016 \\
1233 \\
1481 \\
1763\end{array}$ \\
\hline $\begin{array}{l}20 \\
21 \\
22 \\
23 \\
24\end{array}$ & $\begin{array}{l}20 \\
21 \\
22 \\
23 \\
24\end{array}$ & $\begin{array}{l}152 \\
169 \\
188 \\
207 \\
228\end{array}$ & $\begin{array}{l}400 \\
441 \\
484 \\
529 \\
576\end{array}$ & $\begin{array}{l}2080 \\
2436 \\
2832 \\
3273 \\
3760\end{array}$ \\
\hline $\begin{array}{l}25 \\
26 \\
27 \\
28 \\
29\end{array}$ & $\begin{array}{l}25 \\
26 \\
27 \\
28 \\
29\end{array}$ & $\begin{array}{l}250 \\
273 \\
297 \\
323 \\
350\end{array}$ & $\begin{array}{l}625 \\
676 \\
729 \\
784 \\
841\end{array}$ & $\begin{array}{l}4297 \\
4886 \\
5531 \\
6234 \\
7000\end{array}$ \\
\hline $\begin{array}{l}30 \\
31 \\
32 \\
33 \\
34\end{array}$ & $\begin{array}{l}30 \\
31 \\
32 \\
33 \\
34\end{array}$ & $\begin{array}{l}378 \\
407 \\
438 \\
470 \\
504\end{array}$ & $\begin{array}{r}900 \\
961 \\
1024 \\
1089 \\
1156\end{array}$ & $\begin{array}{r}7830 \\
8729 \\
9699 \\
10745 \\
11870\end{array}$ \\
\hline $\begin{array}{l}35 \\
36 \\
37 \\
38 \\
39\end{array}$ & $\begin{array}{l}35 \\
36 \\
37 \\
38 \\
39\end{array}$ & $\begin{array}{l}539 \\
575 \\
613 \\
653 \\
694\end{array}$ & $\begin{array}{l}1225 \\
1296 \\
1369 \\
1444 \\
1521\end{array}$ & $\begin{array}{l}13077 \\
14370 \\
15753 \\
17230 \\
18804\end{array}$ \\
\hline
\end{tabular}

in the ranges of temperature from $0^{\circ}$ to $190^{\circ} \mathrm{C}$ and $0^{\circ}$ to $-190^{\circ} \mathrm{C}$, respectively

See footnote at end of table. 
TABLE 11.-Data for computing temperatures in platinum resistance thermometry by the equations ${ }^{1}$ - Continued

\begin{tabular}{|c|c|c|c|c|}
\hline$-t$ & $-\frac{t}{10^{6}}$ & $-(4 t-300) \frac{t^{2}}{10^{12}}$ & $\frac{t^{2}}{10^{7}}$ & $(3 t-200) \frac{t^{3}}{10^{12}}$ \\
\hline $\begin{array}{l}40 \\
41 \\
42 \\
43 \\
44\end{array}$ & $\begin{array}{l}40 \\
41 \\
42 \\
43 \\
44\end{array}$ & $\begin{array}{l}736 \\
780 \\
826 \\
873 \\
922\end{array}$ & $\begin{array}{l}1600 \\
1681 \\
1764 \\
1849 \\
1936\end{array}$ & $\begin{array}{l}20480 \\
22261 \\
24153 \\
26158 \\
28281\end{array}$ \\
\hline $\begin{array}{l}45 \\
46 \\
47 \\
48 \\
49\end{array}$ & $\begin{array}{l}45 \\
46 \\
47 \\
48 \\
49\end{array}$ & $\begin{array}{r}972 \\
1024 \\
1078 \\
1134 \\
1191\end{array}$ & $\begin{array}{l}2025 \\
2116 \\
2209 \\
2304 \\
2401\end{array}$ & $\begin{array}{l}30527 \\
32900 \\
35404 \\
38044 \\
40824\end{array}$ \\
\hline $\begin{array}{l}50 \\
51 \\
52 \\
53 \\
54\end{array}$ & $\begin{array}{l}50 \\
51 \\
52 \\
53 \\
54\end{array}$ & $\begin{array}{l}1250 \\
1311 \\
1374 \\
1438 \\
1505\end{array}$ & $\begin{array}{l}2500 \\
2601 \\
2704 \\
2809 \\
2916\end{array}$ & $\begin{array}{l}43750 \\
46826 \\
50056 \\
53447 \\
57002\end{array}$ \\
\hline $\begin{array}{l}55 \\
56 \\
57 \\
58 \\
59\end{array}$ & $\begin{array}{l}55 \\
56 \\
57 \\
58 \\
59\end{array}$ & $\begin{array}{l}1573 \\
1643 \\
1715 \\
1790 \\
1866\end{array}$ & $\begin{array}{l}3025 \\
3136 \\
3249 \\
3364 \\
3481\end{array}$ & $\begin{array}{l}60727 \\
64627 \\
68707 \\
72972 \\
77428\end{array}$ \\
\hline $\begin{array}{l}60 \\
61 \\
62 \\
63 \\
64\end{array}$ & $\begin{array}{l}60 \\
61 \\
62 \\
63 \\
64\end{array}$ & $\begin{array}{l}1944 \\
2024 \\
2107 \\
2191 \\
2277\end{array}$ & $\begin{array}{l}3600 \\
3721 \\
3844 \\
3969 \\
4096\end{array}$ & $\begin{array}{r}82080 \\
86934 \\
91995 \\
97268 \\
102760\end{array}$ \\
\hline $\begin{array}{l}65 \\
66 \\
67 \\
68 \\
69\end{array}$ & $\begin{array}{l}65 \\
66 \\
67 \\
68 \\
69\end{array}$ & $\begin{array}{l}2366 \\
2457 \\
2550 \\
2645 \\
2742\end{array}$ & $\begin{array}{l}4225 \\
4356 \\
4489 \\
4624 \\
4761\end{array}$ & $\begin{array}{l}108477 \\
114423 \\
120606 \\
127031 \\
133703\end{array}$ \\
\hline $\begin{array}{l}70 \\
71 \\
72 \\
73 \\
74\end{array}$ & $\begin{array}{l}70 \\
71 \\
72 \\
73 \\
74\end{array}$ & $\begin{array}{l}2842 \\
2944 \\
3048 \\
3155 \\
3264\end{array}$ & $\begin{array}{l}4900 \\
5041 \\
5184 \\
5329 \\
5476\end{array}$ & $\begin{array}{l}140630 \\
147817 \\
155271 \\
162998 \\
171005\end{array}$ \\
\hline $\begin{array}{l}75 \\
76 \\
77 \\
78 \\
79\end{array}$ & $\begin{array}{l}75 \\
76 \\
77 \\
78 \\
79\end{array}$ & $\begin{array}{l}3375 \\
3489 \\
3605 \\
3723 \\
3844\end{array}$ & $\begin{array}{l}5625 \\
5776 \\
5929 \\
6084 \\
6241\end{array}$ & $\begin{array}{l}179297 \\
187882 \\
196766 \\
205956 \\
215458\end{array}$ \\
\hline $\begin{array}{l}80 \\
81 \\
82 \\
83 \\
84\end{array}$ & $\begin{array}{l}80 \\
81 \\
82 \\
83 \\
84\end{array}$ & $\begin{array}{l}3968 \\
4094 \\
4223 \\
4354 \\
4488\end{array}$ & $\begin{array}{l}6400 \\
6561 \\
6724 \\
6889 \\
7056\end{array}$ & $\begin{array}{l}225280 \\
235428 \\
245910 \\
256732 \\
267902\end{array}$ \\
\hline $\begin{array}{l}85 \\
86 \\
87 \\
88 \\
89\end{array}$ & $\begin{array}{l}85 \\
86 \\
87 \\
88 \\
89\end{array}$ & $\begin{array}{l}4624 \\
4763 \\
4905 \\
5049 \\
5196\end{array}$ & $\begin{array}{l}7225 \\
7396 \\
7569 \\
7744 \\
7921\end{array}$ & $\begin{array}{l}279427 \\
291314 \\
303570 \\
316203 \\
329221\end{array}$ \\
\hline
\end{tabular}

${ }^{1}$ See footnote at end of table. 
366 Journal of Research of the National Bureau of Standards

TABLE 11.-Data for computing temperatures in platinum resistance thermometry by the equations ${ }^{1}$ - Continued

\begin{tabular}{|c|c|c|c|c|}
\hline$-t$ & $-\frac{t}{10^{6}}$ & $-(4 t-300) \frac{t^{2}}{10^{12}}$ & $\frac{t^{2}}{10^{7}}$ & $(3 t-200) \frac{t^{3}}{10^{12}}$ \\
\hline $\begin{array}{l}90 \\
91 \\
92 \\
93 \\
94\end{array}$ & $\begin{array}{l}90 \\
91 \\
92 \\
93 \\
94\end{array}$ & $\begin{array}{l}5346 \\
5499 \\
5654 \\
5812 \\
5973\end{array}$ & $\begin{array}{l}8100 \\
8281 \\
8464 \\
8649 \\
8836\end{array}$ & $\begin{array}{l}342630 \\
356439 \\
370655 \\
385287 \\
400341\end{array}$ \\
\hline $\begin{array}{l}95 \\
96 \\
97 \\
98 \\
99\end{array}$ & $\begin{array}{l}95 \\
96 \\
97 \\
98 \\
99\end{array}$ & $\begin{array}{l}6137 \\
6304 \\
6473 \\
6646 \\
6821\end{array}$ & $\begin{array}{l}9025 \\
9216 \\
9409 \\
9604 \\
9801\end{array}$ & $\begin{array}{l}415827 \\
431751 \\
448122 \\
464949 \\
482239\end{array}$ \\
\hline $\begin{array}{l}100 \\
101 \\
102 \\
103 \\
104\end{array}$ & $\begin{array}{l}100 \\
101 \\
102 \\
103 \\
104\end{array}$ & $\begin{array}{l}7000 \\
7182 \\
7366 \\
7554 \\
7744\end{array}$ & $\begin{array}{l}10000 \\
10201 \\
10404 \\
10609 \\
10816\end{array}$ & $\begin{array}{l}500000 \\
518241 \\
536971 \\
556198 \\
575930\end{array}$ \\
\hline $\begin{array}{l}105 \\
106 \\
107 \\
108 \\
109\end{array}$ & $\begin{array}{l}105 \\
106 \\
107 \\
108 \\
109\end{array}$ & $\begin{array}{l}7938 \\
8135 \\
8335 \\
8538 \\
8744\end{array}$ & $\begin{array}{l}11025 \\
11236 \\
11449 \\
11664 \\
11881\end{array}$ & $\begin{array}{l}596177 \\
616946 \\
638247 \\
660089 \\
682480\end{array}$ \\
\hline $\begin{array}{l}110 \\
111 \\
112 \\
113 \\
114\end{array}$ & $\begin{array}{l}110 \\
111 \\
112 \\
113 \\
114\end{array}$ & $\begin{array}{l}8954 \\
9167 \\
9383 \\
9602 \\
9825\end{array}$ & $\begin{array}{l}12100 \\
12321 \\
12544 \\
12769 \\
12996\end{array}$ & $\begin{array}{l}705430 \\
728947 \\
753041 \\
777721 \\
802997\end{array}$ \\
\hline $\begin{array}{l}115 \\
116 \\
117 \\
118 \\
119\end{array}$ & $\begin{array}{l}115 \\
116 \\
117 \\
118 \\
119\end{array}$ & $\begin{array}{l}10051 \\
10280 \\
10513 \\
10749 \\
10989\end{array}$ & $\begin{array}{l}13225 \\
13456 \\
13689 \\
13924 \\
14161\end{array}$ & $\begin{array}{l}828877 \\
855371 \\
882489 \\
910240 \\
938634\end{array}$ \\
\hline $\begin{array}{l}120 \\
121 \\
122 \\
123 \\
124\end{array}$ & $\begin{array}{l}120 \\
121 \\
122 \\
123 \\
124\end{array}$ & $\begin{array}{l}11232 \\
11479 \\
11729 \\
11982 \\
12239\end{array}$ & $\begin{array}{l}14400 \\
14641 \\
14884 \\
15129 \\
15376\end{array}$ & $\begin{array}{r}967680 \\
997389 \\
1027770 \\
1058833 \\
1090589\end{array}$ \\
\hline $\begin{array}{l}125 \\
126 \\
127 \\
128 \\
129\end{array}$ & $\begin{array}{l}125 \\
126 \\
127 \\
128 \\
129\end{array}$ & $\begin{array}{l}12500 \\
12764 \\
13032 \\
13304 \\
13579\end{array}$ & $\begin{array}{l}15625 \\
15876 \\
16129 \\
16384 \\
16641\end{array}$ & $\begin{array}{l}1123047 \\
1156217 \\
1190111 \\
1224737 \\
1260106\end{array}$ \\
\hline $\begin{array}{l}130 \\
131 \\
132 \\
133 \\
134\end{array}$ & $\begin{array}{l}130 \\
131 \\
132 \\
133 \\
134\end{array}$ & $\begin{array}{l}13858 \\
14141 \\
14427 \\
14717 \\
15011\end{array}$ & $\begin{array}{l}16900 \\
17161 \\
17424 \\
17689 \\
17956\end{array}$ & $\begin{array}{l}1296230 \\
1333118 \\
1370781 \\
1409230 \\
1448475\end{array}$ \\
\hline $\begin{array}{l}135 \\
136 \\
137 \\
138 \\
139\end{array}$ & $\begin{array}{l}135 \\
136 \\
137 \\
138 \\
139\end{array}$ & $\begin{array}{l}15309 \\
15611 \\
15916 \\
16225 \\
16539\end{array}$ & $\begin{array}{l}18225 \\
18496 \\
18769 \\
19044 \\
19321\end{array}$ & $\begin{array}{l}1488527 \\
1529397 \\
1571097 \\
1613636 \\
1657027\end{array}$ \\
\hline
\end{tabular}

1 See footnote at end of table. 
TABLE 11.-Data for computing temperatures in platinum resistance thermometry by the equations ${ }^{1}$-Continued

\begin{tabular}{|c|c|c|c|c|}
\hline$-t$ & $-\frac{t}{10^{6}}$ & $-(4 t-300) \frac{t^{2}}{10^{12}}$ & $\frac{t^{2}}{10^{7}}$ & $(3 t-200) \frac{t^{3}}{10^{12}}$ \\
\hline $\begin{array}{l}140 \\
141 \\
142 \\
143 \\
144\end{array}$ & $\begin{array}{l}140 \\
141 \\
142 \\
143 \\
144\end{array}$ & $\begin{array}{l}16856 \\
17177 \\
17502 \\
17832 \\
18165\end{array}$ & $\begin{array}{l}19600 \\
19881 \\
20164 \\
20449 \\
20736\end{array}$ & $\begin{array}{l}1701280 \\
1746407 \\
1792418 \\
1839326 \\
1887142\end{array}$ \\
\hline $\begin{array}{l}145 \\
146 \\
147 \\
148 \\
149\end{array}$ & $\begin{array}{l}145 \\
146 \\
147 \\
148 \\
149\end{array}$ & $\begin{array}{l}18502 \\
18843 \\
19189 \\
19538 \\
19892\end{array}$ & $\begin{array}{l}21025 \\
21316 \\
21609 \\
21904 \\
22201\end{array}$ & $\begin{array}{l}1935877 \\
1985543 \\
2036151 \\
2087714 \\
2140243\end{array}$ \\
\hline $\begin{array}{l}150 \\
151 \\
152 \\
153 \\
154\end{array}$ & $\begin{array}{l}150 \\
151 \\
152 \\
153 \\
154\end{array}$ & $\begin{array}{l}20250 \\
20612 \\
20978 \\
21349 \\
21724\end{array}$ & $\begin{array}{l}22500 \\
22801 \\
23104 \\
23409 \\
23716\end{array}$ & $\begin{array}{l}2193750 \\
2248247 \\
2303746 \\
2360259 \\
2417799\end{array}$ \\
\hline $\begin{array}{l}155 \\
156 \\
157 \\
158 \\
159\end{array}$ & $\begin{array}{l}155 \\
156 \\
157 \\
158 \\
159\end{array}$ & $\begin{array}{l}22103 \\
22486 \\
22874 \\
23266 \\
23663\end{array}$ & $\begin{array}{l}24025 \\
24336 \\
24649 \\
24964 \\
25281\end{array}$ & $\begin{array}{l}2476377 \\
2536006 \\
2596698 \\
2658466 \\
2721323\end{array}$ \\
\hline $\begin{array}{l}160 \\
161 \\
162 \\
163 \\
164\end{array}$ & $\begin{array}{l}160 \\
161 \\
162 \\
163 \\
164\end{array}$ & $\begin{array}{l}24064 \\
24469 \\
24879 \\
25294 \\
25713\end{array}$ & $\begin{array}{l}25600 \\
25921 \\
26244 \\
26569 \\
26896\end{array}$ & $\begin{array}{l}2785280 \\
2850351 \\
2916548 \\
2983885 \\
3052373\end{array}$ \\
\hline $\begin{array}{l}165 \\
166 \\
167 \\
168 \\
169\end{array}$ & $\begin{array}{l}165 \\
166 \\
167 \\
168 \\
169\end{array}$ & $\begin{array}{l}26136 \\
26564 \\
26997 \\
27434 \\
27876\end{array}$ & $\begin{array}{l}27225 \\
27556 \\
27889 \\
28224 \\
28561\end{array}$ & $\begin{array}{l}3122027 \\
3192859 \\
3264882 \\
3338109 \\
3412554\end{array}$ \\
\hline $\begin{array}{l}170 \\
171 \\
172 \\
173 \\
174\end{array}$ & $\begin{array}{l}170 \\
171 \\
172 \\
173 \\
174\end{array}$ & $\begin{array}{l}28322 \\
28773 \\
29229 \\
29690 \\
30155\end{array}$ & $\begin{array}{l}28900 \\
29241 \\
29584 \\
29929 \\
30276\end{array}$ & $\begin{array}{l}3488230 \\
3565150 \\
3643329 \\
3722779 \\
3803513\end{array}$ \\
\hline $\begin{array}{l}175 \\
176 \\
177 \\
178 \\
179\end{array}$ & $\begin{array}{l}175 \\
176 \\
177 \\
178 \\
179\end{array}$ & $\begin{array}{l}30625 \\
31100 \\
31580 \\
32064 \\
32554\end{array}$ & $\begin{array}{l}30625 \\
30976 \\
31329 \\
31684 \\
32041\end{array}$ & $\begin{array}{l}3885547 \\
3968893 \\
4053565 \\
4139578 \\
4226945\end{array}$ \\
\hline $\begin{array}{l}180 \\
181 \\
182 \\
183 \\
184\end{array}$ & $\begin{array}{l}180 \\
181 \\
182 \\
183 \\
184\end{array}$ & $\begin{array}{l}33048 \\
33547 \\
34051 \\
34561 \\
35075\end{array}$ & $\begin{array}{l}32400 \\
32761 \\
33124 \\
33489 \\
33856\end{array}$ & $\begin{array}{l}4315680 \\
4405798 \\
4497312 \\
4590237 \\
4684587\end{array}$ \\
\hline $\begin{array}{l}185 \\
186 \\
187 \\
188 \\
189\end{array}$ & $\begin{array}{l}185 \\
186 \\
187 \\
188 \\
189\end{array}$ & $\begin{array}{l}35594 \\
36118 \\
36648 \\
37182 \\
37721\end{array}$ & $\begin{array}{l}34225 \\
34596 \\
34969 \\
35344 \\
35721\end{array}$ & $\begin{array}{l}4780377 \\
4877621 \\
4976333 \\
5076529 \\
5178223\end{array}$ \\
\hline 190 & 0.000190 & 0.000038266 & 0.0036100 & 0.005281430 \\
\hline
\end{tabular}

1 The constants $R_{0}, A, B$, and $C$ are determined by measurements of resistances at the melting point of ice, the boiling points of water, sulfur, and oxygen, respectively. The powers of 10 used in the computations as coefficients of the functions of $t_{1}$ and of the constants $B$ and $C$ are such as to make the order of magnitude of the constant factors greater than 1 but less than 10 and to effect a simple regulation of decimal points. 
368 Journal of Research of the National Bureau of Standards

TABLE 12.-Data for computing temperatures in platinum resistance thermometry by the equations ${ }^{1}$

$$
t_{2}=\frac{\left[\left(R_{t} / R_{0}\right)-1\right]+B t_{1}^{2}}{A+2 B t_{1}}
$$

and

$$
t_{2}=\frac{\left[\left(R_{t} / R_{0}\right)-1\right]+B t_{1}^{2}+E\left[\left(t_{1} / 100\right)-1\right] t_{1}^{3}}{A+2 B t_{1}+F\left[\left(t_{1} / 100\right)-1\right] t_{1}^{2}}
$$

\begin{tabular}{|c|c|c|c|c|}
\hline$-t$ & $-\frac{t}{10^{6}}$ & $-\left(\frac{t}{100}-1\right) \frac{t^{2}}{10^{9}}$ & $\frac{t^{2}}{2 \times 10^{6}}$ & $\left(\frac{t}{100}-1\right) \frac{t^{3}}{10^{9}}$ \\
\hline $\begin{array}{l}0 \\
1 \\
2 \\
3 \\
4\end{array}$ & $\begin{array}{r}0.000000 \\
1 \\
2 \\
3 \\
4\end{array}$ & $\begin{array}{r}0.000000000 \\
1 \\
4 \\
9 \\
17\end{array}$ & $\begin{array}{r}0.0000000 \\
5 \\
20 \\
45 \\
80\end{array}$ & $\begin{array}{r}0.000000000 \\
1 \\
8 \\
28 \\
67\end{array}$ \\
\hline $\begin{array}{l}5 \\
6 \\
7 \\
8 \\
9\end{array}$ & $\begin{array}{l}5 \\
6 \\
7 \\
8 \\
9\end{array}$ & $\begin{array}{l}26 \\
38 \\
52 \\
69 \\
88\end{array}$ & $\begin{array}{l}125 \\
180 \\
245 \\
320 \\
405\end{array}$ & $\begin{array}{l}131 \\
229 \\
367 \\
553 \\
795\end{array}$ \\
\hline $\begin{array}{l}10 \\
11 \\
12 \\
13 \\
14\end{array}$ & $\begin{array}{l}10 \\
11 \\
12 \\
13 \\
14\end{array}$ & $\begin{array}{l}110 \\
134 \\
161 \\
191 \\
223\end{array}$ & $\begin{array}{l}500 \\
605 \\
720 \\
845 \\
980\end{array}$ & $\begin{array}{l}1100 \\
1477 \\
1935 \\
2483 \\
3128\end{array}$ \\
\hline $\begin{array}{l}15 \\
16 \\
17 \\
18 \\
19\end{array}$ & $\begin{array}{l}15 \\
16 \\
17 \\
18 \\
19\end{array}$ & $\begin{array}{l}259 \\
297 \\
338 \\
382 \\
430\end{array}$ & $\begin{array}{l}1125 \\
1280 \\
1445 \\
1620 \\
1805\end{array}$ & $\begin{array}{l}3881 \\
4751 \\
5748 \\
6882 \\
8162\end{array}$ \\
\hline $\begin{array}{l}20 \\
21 \\
22 \\
23 \\
24\end{array}$ & $\begin{array}{l}20 \\
21 \\
22 \\
23 \\
24\end{array}$ & $\begin{array}{l}480 \\
534 \\
590 \\
651 \\
714\end{array}$ & $\begin{array}{l}2000 \\
2205 \\
2420 \\
2645 \\
2880\end{array}$ & $\begin{array}{r}9600 \\
11206 \\
12991 \\
14965 \\
17142\end{array}$ \\
\hline $\begin{array}{l}25 \\
26 \\
27 \\
28 \\
29\end{array}$ & $\begin{array}{l}25 \\
26 \\
27 \\
28 \\
29\end{array}$ & $\begin{array}{r}781 \\
852 \\
926 \\
1004 \\
1085\end{array}$ & $\begin{array}{l}3125 \\
3380 \\
3645 \\
3920 \\
4205\end{array}$ & $\begin{array}{l}19531 \\
22146 \\
24997 \\
28099 \\
31462\end{array}$ \\
\hline $\begin{array}{l}30 \\
31 \\
32 \\
33 \\
34\end{array}$ & $\begin{array}{l}30 \\
31 \\
32 \\
33 \\
34\end{array}$ & $\begin{array}{l}1170 \\
1259 \\
1352 \\
1448 \\
1549\end{array}$ & $\begin{array}{l}4500 \\
4805 \\
5120 \\
5445 \\
5780\end{array}$ & $\begin{array}{l}35100 \\
39026 \\
43254 \\
47796 \\
52667\end{array}$ \\
\hline $\begin{array}{l}35 \\
36 \\
37 \\
38 \\
39\end{array}$ & $\begin{array}{l}35 \\
36 \\
37 \\
38 \\
39\end{array}$ & $\begin{array}{l}1654 \\
1763 \\
1876 \\
1993 \\
2114\end{array}$ & $\begin{array}{l}6125 \\
6480 \\
6845 \\
7220 \\
7605\end{array}$ & $\begin{array}{l}57881 \\
63452 \\
69395 \\
75723 \\
82453\end{array}$ \\
\hline
\end{tabular}

in the ranges of temperature from $0^{\circ}$ to $190^{\circ} \mathrm{C}$ and $0^{\circ}$ to $-190^{\circ} \mathrm{C}$, respectively.

${ }^{1}$ See footnote at end of table. 
TABLE 12.-Data for computing temperatures in platinum resistance thermometry by the equations ${ }^{1}$-Continued

\begin{tabular}{|c|c|c|c|c|}
\hline$-t$ & $-\frac{t}{10^{6}}$ & $-\left(\frac{t}{100}-1\right) \frac{t^{2}}{10^{9}}$ & $\frac{t^{2}}{2 \times 10^{6}}$ & $\left(\frac{t}{100}-1\right) \frac{t^{3}}{10^{9}}$ \\
\hline $\begin{array}{l}40 \\
41 \\
42 \\
43 \\
44\end{array}$ & $\begin{array}{l}40 \\
41 \\
42 \\
43 \\
44\end{array}$ & $\begin{array}{l}2240 \\
2370 \\
2505 \\
2644 \\
2788\end{array}$ & $\begin{array}{l}8000 \\
8405 \\
8820 \\
9245 \\
9680\end{array}$ & $\begin{array}{r}89600 \\
97179 \\
105205 \\
113695 \\
122665\end{array}$ \\
\hline $\begin{array}{l}45 \\
46 \\
47 \\
48 \\
49\end{array}$ & $\begin{array}{l}45 \\
46 \\
47 \\
48 \\
49\end{array}$ & $\begin{array}{l}2936 \\
3089 \\
3247 \\
3410 \\
3577\end{array}$ & $\begin{array}{l}10125 \\
10580 \\
11045 \\
11520 \\
12005\end{array}$ & $\begin{array}{l}132131 \\
142111 \\
152620 \\
163676 \\
175297\end{array}$ \\
\hline $\begin{array}{l}50 \\
51 \\
52 \\
53 \\
54\end{array}$ & $\begin{array}{l}50 \\
51 \\
52 \\
53 \\
54\end{array}$ & $\begin{array}{l}3750 \\
3928 \\
4110 \\
4298 \\
4491\end{array}$ & $\begin{array}{l}12500 \\
13005 \\
13520 \\
14045 \\
14580\end{array}$ & $\begin{array}{l}187500 \\
200303 \\
213724 \\
227782 \\
242495\end{array}$ \\
\hline $\begin{array}{l}55 \\
56 \\
57 \\
58 \\
59\end{array}$ & $\begin{array}{l}55 \\
56 \\
57 \\
58 \\
59\end{array}$ & $\begin{array}{l}4689 \\
4892 \\
5101 \\
5315 \\
5535\end{array}$ & $\begin{array}{l}15125 \\
15680 \\
16245 \\
16820 \\
17405\end{array}$ & $\begin{array}{l}257881 \\
273961 \\
290753 \\
308277 \\
326553\end{array}$ \\
\hline $\begin{array}{l}60 \\
61 \\
62 \\
63 \\
64\end{array}$ & $\begin{array}{l}60 \\
61 \\
62 \\
63 \\
64\end{array}$ & $\begin{array}{l}5760 \\
5991 \\
6227 \\
6469 \\
6717\end{array}$ & $\begin{array}{l}18000 \\
18605 \\
19220 \\
19845 \\
20480\end{array}$ & $\begin{array}{l}345600 \\
365439 \\
386091 \\
407577 \\
429916\end{array}$ \\
\hline $\begin{array}{l}65 \\
66 \\
67 \\
68 \\
69\end{array}$ & $\begin{array}{l}65 \\
66 \\
67 \\
68 \\
69\end{array}$ & $\begin{array}{l}6971 \\
7231 \\
7497 \\
7768 \\
8046\end{array}$ & $\begin{array}{l}21125 \\
21780 \\
22445 \\
23120 \\
23805\end{array}$ & $\begin{array}{l}453131 \\
477243 \\
502274 \\
528246 \\
555180\end{array}$ \\
\hline $\begin{array}{l}70 \\
71 \\
72 \\
73 \\
74\end{array}$ & $\begin{array}{l}70 \\
71 \\
72 \\
73 \\
74\end{array}$ & $\begin{array}{l}8330 \\
8620 \\
8916 \\
9219 \\
9528\end{array}$ & $\begin{array}{l}24500 \\
25205 \\
25920 \\
26645 \\
27380\end{array}$ & $\begin{array}{l}583100 \\
612028 \\
641987 \\
672999 \\
705090\end{array}$ \\
\hline $\begin{array}{l}75 \\
76 \\
77 \\
78 \\
79\end{array}$ & $\begin{array}{l}75 \\
76 \\
77 \\
78 \\
79\end{array}$ & $\begin{array}{r}9844 \\
10166 \\
10494 \\
10830 \\
11171\end{array}$ & $\begin{array}{l}28125 \\
28880 \\
29645 \\
30420 \\
31205\end{array}$ & $\begin{array}{l}738281 \\
772598 \\
808063 \\
844703 \\
882540\end{array}$ \\
\hline $\begin{array}{l}80 \\
81 \\
82 \\
83 \\
84\end{array}$ & $\begin{array}{l}80 \\
81 \\
82 \\
83 \\
84\end{array}$ & $\begin{array}{l}11520 \\
11875 \\
12238 \\
12607 \\
12983\end{array}$ & $\begin{array}{l}32000 \\
32805 \\
33620 \\
34445 \\
35280\end{array}$ & $\begin{array}{r}921600 \\
961908 \\
1003490 \\
1046370 \\
1090575\end{array}$ \\
\hline $\begin{array}{l}85 \\
86 \\
87 \\
88 \\
89\end{array}$ & $\begin{array}{l}85 \\
86 \\
87 \\
88 \\
89\end{array}$ & $\begin{array}{l}13366 \\
13757 \\
14154 \\
14559 \\
14971\end{array}$ & $\begin{array}{l}36125 \\
36980 \\
37845 \\
38720 \\
39605\end{array}$ & $\begin{array}{l}1136131 \\
1183064 \\
1231401 \\
1281167 \\
1332391\end{array}$ \\
\hline
\end{tabular}

1 See footnote at end of table. 
TABLE 12.-Data for computing temperatures in platinum resistance thermometry by the equations ${ }^{1}$ - Continued

\begin{tabular}{|c|c|c|c|c|}
\hline$-t$ & $-\frac{t}{10^{6}}$ & $-\left(\frac{t}{100}-1\right) \frac{t^{2}}{10^{\theta}}$ & $\frac{t^{2}}{2 \times 10^{6}}$ & $\left(\frac{t}{100}-1\right) \frac{t^{3}}{10^{9}}$ \\
\hline $\begin{array}{l}90 \\
91 \\
92 \\
93 \\
94\end{array}$ & $\begin{array}{l}90 \\
91 \\
92 \\
93 \\
94\end{array}$ & $\begin{array}{l}15390 \\
15817 \\
16251 \\
16693 \\
17142\end{array}$ & $\begin{array}{l}40500 \\
41405 \\
42320 \\
43245 \\
44180\end{array}$ & $\begin{array}{l}1385100 \\
1439321 \\
1495081 \\
1552409 \\
1611333\end{array}$ \\
\hline $\begin{array}{r}95 \\
96 \\
97 \\
98 \\
-\quad 99\end{array}$ & $\begin{array}{l}95 \\
96 \\
97 \\
98 \\
99\end{array}$ & $\begin{array}{l}17599 \\
18063 \\
18536 \\
19016 \\
19504\end{array}$ & $\begin{array}{l}45125 \\
46080 \\
47045 \\
48020 \\
49005\end{array}$ & $\begin{array}{l}1671881 \\
1734083 \\
1797966 \\
1863560 \\
1930895\end{array}$ \\
\hline $\begin{array}{l}100 \\
101 \\
102 \\
103 \\
104\end{array}$ & $\begin{array}{l}100 \\
101 \\
102 \\
103 \\
104\end{array}$ & $\begin{array}{l}20000 \\
20504 \\
21016 \\
21536 \\
22065\end{array}$ & $\begin{array}{l}50000 \\
51005 \\
52020 \\
53045 \\
54080\end{array}$ & $\begin{array}{l}2000000 \\
2070905 \\
2143640 \\
2218236 \\
2294723\end{array}$ \\
\hline $\begin{array}{l}105 \\
106 \\
107 \\
108 \\
109\end{array}$ & $\begin{array}{l}105 \\
106 \\
107 \\
108 \\
109\end{array}$ & $\begin{array}{l}22601 \\
23146 \\
23699 \\
24261 \\
24831\end{array}$ & $\begin{array}{l}55125 \\
56180 \\
57245 \\
58320 \\
59405\end{array}$ & $\begin{array}{l}2373131 \\
2453493 \\
2535839 \\
2620201 \\
2706611\end{array}$ \\
\hline $\begin{array}{l}110 \\
111 \\
112 \\
113 \\
114\end{array}$ & $\begin{array}{l}110 \\
111 \\
112 \\
113 \\
114\end{array}$ & $\begin{array}{l}25410 \\
25997 \\
26593 \\
27198 \\
27811\end{array}$ & $\begin{array}{l}60500 \\
61605 \\
62720 \\
63845 \\
64980\end{array}$ & $\begin{array}{l}2795100 \\
2885701 \\
2978447 \\
3073371 \\
3170504\end{array}$ \\
\hline $\begin{array}{l}115 \\
116 \\
117 \\
118 \\
119\end{array}$ & $\begin{array}{l}115 \\
116 \\
117 \\
118 \\
119\end{array}$ & $\begin{array}{l}28434 \\
29065 \\
29705 \\
30354 \\
31013\end{array}$ & $\begin{array}{l}66125 \\
67280 \\
68445 \\
69620 \\
70805\end{array}$ & $\begin{array}{l}3269881 \\
3371535 \\
3475500 \\
3581810 \\
3690498\end{array}$ \\
\hline $\begin{array}{l}120 \\
121 \\
122 \\
123 \\
124\end{array}$ & $\begin{array}{l}120 \\
121 \\
122 \\
123 \\
124\end{array}$ & $\begin{array}{l}31680 \\
32357 \\
33042 \\
33738 \\
34442\end{array}$ & $\begin{array}{r}72000 \\
73205 \\
74420 \\
75645 \\
76880\end{array}$ & $\begin{array}{l}3801600 \\
3915150 \\
4031183 \\
4149733 \\
4270838\end{array}$ \\
\hline $\begin{array}{l}125 \\
126 \\
127 \\
128 \\
129\end{array}$ & $\begin{array}{l}125 \\
126 \\
127 \\
128 \\
129\end{array}$ & $\begin{array}{l}35156 \\
35880 \\
36613 \\
37356 \\
38108\end{array}$ & $\begin{array}{l}78125 \\
79380 \\
80645 \\
81920 \\
83205\end{array}$ & $\begin{array}{l}4394531 \\
4520850 \\
4649829 \\
4781507 \\
4915918\end{array}$ \\
\hline $\begin{array}{l}130 \\
131 \\
132 \\
133 \\
134\end{array}$ & $\begin{array}{l}130 \\
131 \\
132 \\
133 \\
134\end{array}$ & $\begin{array}{l}38870 \\
39642 \\
40424 \\
41215 \\
42017\end{array}$ & $\begin{array}{l}84500 \\
85805 \\
87120 \\
88445 \\
89780\end{array}$ & $\begin{array}{l}5053100 \\
5193090 \\
5335926 \\
5481644 \\
5630283\end{array}$ \\
\hline $\begin{array}{l}135 \\
136 \\
137 \\
138 \\
139\end{array}$ & $\begin{array}{l}135 \\
136 \\
137 \\
138 \\
139\end{array}$ & $\begin{array}{l}42829 \\
43651 \\
44483 \\
45325 \\
46177\end{array}$ & $\begin{array}{l}91125 \\
92480 \\
93845 \\
95220 \\
96605\end{array}$ & $\begin{array}{l}5781881 \\
5936476 \\
6094107 \\
6254811 \\
6418629\end{array}$ \\
\hline
\end{tabular}

\footnotetext{
See footnote at end of table.
} 
TABLE 12.-Data for computing temperatures in platinum resistance thermometry by the equations ${ }^{1}$ - Continued

\begin{tabular}{|c|c|c|c|c|}
\hline$-t$ & $-\frac{t}{10^{6}}$ & $-\left(\frac{t}{100}-1\right) \frac{t^{2}}{10^{9}}$ & $\frac{t^{2}}{2 \times 10^{6}}$ & $\left(\frac{t}{100}-1\right) \frac{t^{3}}{10^{9}}$ \\
\hline $\begin{array}{l}140 \\
141 \\
142 \\
143 \\
144\end{array}$ & $\begin{array}{l}140 \\
141 \\
142 \\
143 \\
144\end{array}$ & $\begin{array}{l}47040 \\
47913 \\
48797 \\
49691 \\
50596\end{array}$ & $\begin{array}{r}98000 \\
99405 \\
100820 \\
102245 \\
103680\end{array}$ & $\begin{array}{l}6585600 \\
6755763 \\
6929157 \\
7105823 \\
7285801\end{array}$ \\
\hline $\begin{array}{l}145 \\
146 \\
147 \\
148 \\
149\end{array}$ & $\begin{array}{l}145 \\
146 \\
147 \\
148 \\
149\end{array}$ & $\begin{array}{l}51511 \\
52437 \\
53374 \\
54322 \\
55280\end{array}$ & $\begin{array}{l}105125 \\
106580 \\
108045 \\
109520 \\
111005\end{array}$ & $\begin{array}{l}7469131 \\
7655855 \\
7846012 \\
8039644 \\
8236793\end{array}$ \\
\hline $\begin{array}{l}150 \\
151 \\
152 \\
153 \\
154\end{array}$ & $\begin{array}{l}150 \\
151 \\
152 \\
153 \\
154\end{array}$ & $\begin{array}{l}56250 \\
57231 \\
58222 \\
59225 \\
60239\end{array}$ & $\begin{array}{l}112500 \\
114005 \\
115520 \\
117045 \\
118580\end{array}$ & $\begin{array}{l}8437500 \\
8641807 \\
8849756 \\
9061390 \\
9276751\end{array}$ \\
\hline $\begin{array}{l}155 \\
156 \\
157 \\
158 \\
159\end{array}$ & $\begin{array}{l}155 \\
156 \\
157 \\
158 \\
159\end{array}$ & $\begin{array}{l}61264 \\
62300 \\
63348 \\
64407 \\
65478\end{array}$ & $\begin{array}{l}120125 \\
121680 \\
123245 \\
124820 \\
126405\end{array}$ & $\begin{array}{r}9495881 \\
9718825 \\
9945625 \\
10176325 \\
10410969\end{array}$ \\
\hline $\begin{array}{l}160 \\
161 \\
162 \\
163 \\
164\end{array}$ & $\begin{array}{l}160 \\
161 \\
162 \\
163 \\
164\end{array}$ & $\begin{array}{l}66560 \\
67654 \\
68759 \\
69876 \\
71005\end{array}$ & $\begin{array}{l}128000 \\
129605 \\
131220 \\
132845 \\
134480\end{array}$ & $\begin{array}{l}10649600 \\
10892263 \\
11139003 \\
11389865 \\
11644892\end{array}$ \\
\hline $\begin{array}{l}165 \\
166 \\
167 \\
168 \\
169\end{array}$ & $\begin{array}{l}165 \\
166 \\
167 \\
168 \\
169\end{array}$ & $\begin{array}{l}72146 \\
73299 \\
74464 \\
75640 \\
76829\end{array}$ & $\begin{array}{l}136125 \\
137780 \\
139445 \\
141120 \\
142805\end{array}$ & $\begin{array}{l}11904131 \\
12167627 \\
12435426 \\
12707574 \\
12984116\end{array}$ \\
\hline $\begin{array}{l}170 \\
171 \\
172 \\
173 \\
174\end{array}$ & $\begin{array}{l}170 \\
171 \\
172 \\
173 \\
174\end{array}$ & $\begin{array}{l}78030 \\
79243 \\
80468 \\
81706 \\
82956\end{array}$ & $\begin{array}{l}144500 \\
146205 \\
147920 \\
149645 \\
151380\end{array}$ & $\begin{array}{l}13265100 \\
13550572 \\
13840579 \\
14135167 \\
14434386\end{array}$ \\
\hline $\begin{array}{l}175 \\
176 \\
177 \\
178 \\
179\end{array}$ & $\begin{array}{l}175 \\
176 \\
177 \\
178 \\
179\end{array}$ & $\begin{array}{l}84219 \\
85494 \\
86781 \\
88082 \\
89394\end{array}$ & $\begin{array}{l}153125 \\
154880 \\
156645 \\
158420 \\
160205\end{array}$ & $\begin{array}{l}14738281 \\
15046902 \\
15360295 \\
15678511 \\
16001596\end{array}$ \\
\hline $\begin{array}{l}180 \\
181 \\
182 \\
183 \\
184\end{array}$ & $\begin{array}{l}180 \\
181 \\
182 \\
183 \\
184\end{array}$ & $\begin{array}{l}90720 \\
92058 \\
93410 \\
94774 \\
96151\end{array}$ & $\begin{array}{l}162000 \\
163805 \\
165620 \\
167445 \\
169280\end{array}$ & $\begin{array}{l}16329600 \\
16662572 \\
17000562 \\
17343618 \\
17691791\end{array}$ \\
\hline $\begin{array}{l}185 \\
186 \\
187 \\
188 \\
189\end{array}$ & $\begin{array}{l}185 \\
186 \\
187 \\
188 \\
189\end{array}$ & $\begin{array}{r}97541 \\
98945 \\
100361 \\
101791 \\
103234\end{array}$ & $\begin{array}{l}171125 \\
172980 \\
174845 \\
176720 \\
178605\end{array}$ & $\begin{array}{l}18045131 \\
18403688 \\
18767513 \\
19136655 \\
19511167\end{array}$ \\
\hline 190 & 0.000190 & 0. 000104690 & 0. 0180500 & 0. 019891100 \\
\hline
\end{tabular}

1 The constants $R_{0}, A, B$, and $C$ are determined by measurements of resistances at the melting point of ice, the boiling points of water, sulfur, and oxygen, respectively. $E$ and $F$ are equal to $264 C$ and $364 C$, respectively. The powers of 10 used in the computations as coefficients of the functions of $t_{1}$, and of the constants $B, E$, and $F$ are such as to make the order of magnitude of the constant factors greater than 1 but less than 10 and to effect a simple regulation of decimal points. 
Set decimal point indicators at corresponding positions on the keyboard and middle dial of a calculating machine equivalent to the Marchant Model M. Set the value of $A$ in the keyboard in its proper position for the decimal point and add it into the middle dial. This clears the keyboard. Set the value of $10^{-6} t_{1}$ in the keyboard properly with respect to its decimal point and multiply, by $2 \times 10^{6} B$. This operation adds the product, $2 B t_{1}$, to $A$ in the middle dial. Bring the carriage back to its original position, place the value of $10^{-12}\left(4 t_{1}-\right.$ $300) t_{1}^{2}$ taken from table 11 if equation 12 is used, or the value of $10^{-9}\left[\left(t_{1} / 100\right)-1\right] t_{1}^{2}$ taken from table 12 if equation 14 is used, in the keyboard with proper account of the decimal point, multiply by $10^{12} \mathrm{C}$ for equation 12 , or by $10^{9} \mathrm{~F}$ for equation 14 . This operation adds the third term of the denominator to the sum of the first two and shows the value of the whole denominator in the middle dial. Record this value on paper and clear the dials of the machine. The numerator is next obtained in the same manner but left in the middle dial while the denominator is set in the keyboard. Automatic division now yields the temperature correct to the nearest millidegree. With practice, the computation of a temperature, exclusive of the calculation of $\left(R_{t} / R_{0}\right)-1$, can be made by this procedure in less than 2 minutes.

The powers of 10 used in the computations as coefficients of the functions of $t_{1}$ and of the constants $B$ and $C$ are such as to make the order of magnitude of the constant factors greater than 1 but less than 10 and to effect a simple regulation of decimal points. In table 11, for use with equation 12 , the following are tabulated:

$10^{-6} t_{1}$ to be multiplied by $2 \times 10^{6} B$ to obtain $2 B t_{1}$, as $2 \times 10^{6} B$ is approximately 1.2 .

$10^{-12}\left(4 t_{1}-300\right) t_{1}^{2}$ and $10^{-12}\left(3 t_{1}-200\right) t_{1}^{3}$ to be multiplied by $10^{12} \mathrm{C}$ to obtain $C\left(4 t_{1}-300\right) t_{1}^{2}$ and $C\left(3 t_{1}-200\right) t_{1}^{3}$, since $10^{12} C$ is approximately 4.4 .

$10^{-7} t_{1}^{2}$ to be multiplied by $10^{7} \mathrm{~B}$ to obtain $B t_{1}^{2}$, as $10^{7} \mathrm{~B}$ is approximately 6 .

In table 12, for use with equation 14, the tabulation is analogous. With proper regard for signs, columns 2 and 4 of table 11 or table 12 can be used to calculate temperatures in the upper range from $0^{\circ}$ to $190^{\circ} \mathrm{C}$, if the table for computations of temperatures below $0^{\circ} \mathrm{C}$ is complete. These columns can be extended to $660^{\circ} \mathrm{C}$, or a table of squares can be used.

Computations made with these modified fundamental equations involve the ratio of $R_{t}$ to $R_{0}$, both of which should therefore be measured with the same bridge. This ratio will be practically the same for the same temperature with any calibrated bridge, provided the calibration of a given bridge is consistent, $i$. e., of about the same fractional error in all the dials. The fundamental constants are assumed to be given correctly, i. e., the thermometer must have a proper calibration and this, of course, is true no matter what method of computation is used. The steam point does not appear in the equations and can even be eliminated from the calibration of the thermometer by the use of another fixed point in a suitable region of the temperature scale, such as the freezing point of benzoic acid, or by comparison with a thermometer which has been given a primary calibration.

Washington, December 29, 1944. 